\title{
Management of acute promyelocytic leukemia: recommendations from an expert panel on behalf of the European LeukemiaNet
}

\author{
Miguel A. Sanz, ${ }^{1}$ David Grimwade, ${ }^{2}$ Martin S. Tallman, ${ }^{3}$ Bob Lowenberg, ${ }^{4}$ Pierre Fenaux, ${ }^{5}$ Elihu H. Estey, ${ }^{6}$ Tomoki Naoe, ${ }^{7}$ \\ Eva Lengfelder, ${ }^{8}$ Thomas Büchner, ${ }^{9}$ Hartmut Döhner, ${ }^{10}$ Alan K. Burnett, ${ }^{11}$ and Francesco Lo-Coco ${ }^{12}$ \\ ${ }^{1}$ University Hospital La Fe, Valencia, Spain; ${ }^{2 K i n g}$ 's College London, London, United Kingdom; ${ }^{3}$ Northwestern University, Chicago, IL; ${ }^{4}$ Erasmus University \\ Medical Center, Rotterdam, The Netherlands; ${ }^{5}$ Hopital Avicenne, Paris, France; ${ }^{6}$ Seattle Cancer Care Alliance, WA; ${ }^{7}$ Nagoya University, Nagoya, Japan;

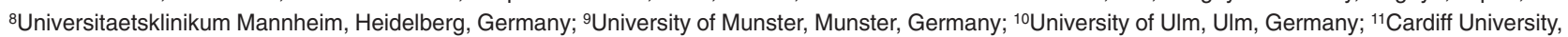 \\ Cardiff, United Kingdom; and ${ }^{12}$ Tor Vergata University, Rome, Italy
}

The introduction of all-trans retinoic acid (ATRA) and, more recently, arsenic trioxide (ATO) into the therapy of acute promyelocytic leukemia (APL) has revolutionized the management and outcome of this disease. Several treatment strategies using these agents, usually in combination with chemotherapy, but also without or with minimal use of cytotoxic agents, have provided excellent therapeutic results. Cure of APL patients, however, is also dependent on peculiar aspects related to the management and supportive measures that are crucial to counteract life-threatening complications associated with the disease biology and molecularly targeted treatment. The European LeukemiaNet recently appointed an international panel of experts to develop evidence- and expert opinion-based guidelines on the diagnosis and management of APL. Together with providing current indications on genetic diagnosis, modern risk-adapted front-line therapy and salvage treatment, the review contains specific recommendations for the identification and management of most important complications such as the bleeding disorder, APL differentiation syndrome, QT prolongation and other ATRAand ATO-related toxicities, as well as for molecular assessment of response to treatment. Finally, the approach to special situations is also discussed, including management of APL in children, elderly patients, and pregnant women. (Blood. 2009;113:1875-1891)

\section{Introduction}

Although the real incidence of acute promyelocytic leukemia (APL) is unknown, it is a relatively rare hematologic malignancy. The number of newly diagnosed cases per year in the United States is estimated to be 600 to $800 .^{1,2}$ One of the most striking features of APL is its age-associated incidence rate. The disease is very uncommon in children less than 10 years of age. Its incidence increases steadily during the teen years, reaches a plateau during early adulthood, and remains constant until it decreases after age 60 years. ${ }^{3}$ This is in marked contrast to other subtypes of acute myeloid leukemia (AML), where there is a steady rise to age 55 years, after which there is an exponential increase. There is also a suggestion in the literature that APL arising as a complication of previous exposure to chemotherapy (particularly drugs targeting topoisomerase II) or radiotherapy is becoming more prevalent, particularly in patients with a history of breast cancer. ${ }^{4-6}$ With respect to the incidence of APL among ethnic groups, contradictory data regarding a presumed higher incidence of APL in persons from Mexico, Central and South America, Italy, and Spain have been reported in the literature. ${ }^{2,7}$ Therefore, this epidemiologic issue is still a matter of controversy and deserves additional investigation.

The introduction of all-trans retinoic acid (ATRA; tretinoin) into the therapy of APL completely revolutionized the management and outcome of this disease. This agent represents one of the most spectacular advances in the treatment of human cancer, providing the first paradigm of molecularly targeted treatment. After the advent of ATRA, the introduction of arsenic trioxide (ATO), probably the most biologically active single drug in APL, has provided a valuable addition to the armamentarium and may have contributed to further improvements in the clinical outcome of this disease. Several treatment strategies using these agents, usually in combination with chemotherapy, have provided excellent therapeutic results with survival rates exceeding $70 \%$ in multicenter clinical trials. Cure of patients with APL depends not only on the effective use of combination therapy involving differentiating and classical cytotoxic agents, but also, critically, upon supportive care measures that take into particular account the biology of the disease and the complications associated with molecularly targeted therapies. Moreover, it is important to consider diagnostic suspicion of APL as a medical emergency (uncommon in AML) that requires several specific and simultaneous actions, including immediate commencement of ATRA therapy, prompt genetic diagnosis, and measures to counteract the coagulopathy.

Although there are some recent exhaustive reviews addressing the management of $\mathrm{APL}^{8-13}$ and national guidelines from the United States and United Kingdom ${ }^{14,15}$ on the management of AML that include some specific items on APL, no comprehensive yet succinct guidelines focused on APL have been produced. Therefore, the European LeukemiaNet appointed an international panel of experts to develop evidence- and expert opinion-based guidelines on the management of APL. The publication of guidelines for the management of challenging malignant diseases provides an excellent tool to spread knowledge of the optimum 
treatment of a given disease, but it is particularly useful in APL for some additional reasons: (1) APL is a rare disease and most patients are treated in institutions with limited experience; (2) the excellent outcome reported recently with current treatments may engender a sense of safety and complacency that may lead to underestimation of the importance of crucial aspects of APL management; and (3) some issues related to the treatment of APL are not well known, lack strong supporting data, and, therefore, involve the risk of adopting erroneous practices, which although appropriate and routinely used for the management of other AML subtypes are inappropriate for management of APL.

\section{Methods}

\subsection{Composition of the panel}

The panel included 12 members with recognized clinical and research expertise in APL, of whom 9 came from European Union countries (France, Germany, Italy, Spain, United Kingdom, and The Netherlands), 2 from the United States, and 1 from Japan.

\subsection{Scope of the review}

A computerized literature search of the PubMed, Cochrane, and Medline databases in the English language was conducted using the key words "acute promyelocytic leukemia" with subheadings "anthracycline," "all-trans retinoic acid," "arsenic trioxide," "retinoic acid/differentiation syndrome," "pregnancy," "disseminated intravascular coagulation" (DIC), "stem cell transplantation," and "febrile neutropenia." Relevant abstracts presented at the 2004, 2005, 2006, and 2007 meetings of the American Society of Hematology and the American Society of Clinical Oncology were also reviewed. The authors have substantial experience in the field. The levels of evidence and grading of recommendations were those defined in the "General Guidelines for Methodologies on Research and Evaluation of Traditional Medicine," 16 which are based on those of the US Agency for Healthcare Research and Quality, formerly the Agency for Health Care Policy and Research (Appendix).

\section{Approach to the patient with suspected APL}

Although there is a general consensus on the need to confirm the diagnosis of APL at the genetic level, both differentiation and supportive therapy should be started before the results of genetic tests are available. In the majority of cases the diagnosis is suggested by the characteristic morphology of the leukemic population, ${ }^{17,18}$ however, it is also important to consider the possibility of APL should any suspicion be raised based on immunophenotypic profile or presence of severe coagulopathy; in all such cases ATRA should be commenced immediately and continued until the diagnosis is confirmed or refuted at the genetic level.

\subsection{Rapid confirmation of genetic diagnosis}

Because the efficacy of differentiation treatment based on retinoids and/or arsenic derivatives is strictly dependent on the presence of the $P M L / R A R A$ fusion in leukemia cells, genetic confirmation of this specific lesion is mandatory in all cases. Morphologic diagnosis of hypergranular (typical) APL is highly predictive of an underlying PML/RARA rearrangement, and immunophenotyping by multiparameter flow cytometry can improve the accuracy of diagnosis, ${ }^{19,20}$ particularly in patients with morphologic features evoking a microgranular (variant) subtype. However, patients with morphologic and/or immunophenotypic features suggestive of APL without the $P M L / R A R A$ rearrangement, and vice versa, have been described in the literature. ${ }^{21-23}$

3.1.1. Diagnostic workup and sample processing. Recommendations for diagnostic workup are summarized in Table 1.

All patients should have a marrow aspirate. This may be omitted only when the peripheral blast count is high and the patient is to be considered for palliative treatment only. A trephine biopsy is required only in the case of a dry marrow aspirate and where no abnormal cells are present in the peripheral blood (PB) to allow a morphologic and molecular diagnosis.

Morphologic studies require a Romanowsky-derived stain, such as Wright, Wright-Giemsa, or May-Grunwald-Giemsa stains, usually complemented by myeloperoxidase or Sudan black B stain. Immunophenotyping by multiparameter flow cytometry can increase the accuracy of a morphologic suspicion of PML/RARApositive APL. Typically, PML/RARA-positive leukemia blasts show immunophenotypic features similar to those of normal promyelocytes $\left(\mathrm{CD} 34^{-/+}\right.$heterogeneous, CD117 ${ }^{-/+} \mathrm{dim}, \mathrm{HLADR}^{-/+} \mathrm{dim}$, $\left.\mathrm{CD} 13^{+/++}, \mathrm{CD} 11 \mathrm{~b}^{-}\right) .{ }^{19}$ However, unlike their normal counterpart, PML/RAR $\alpha$-positive promyelocytes display abnormally low levels of CD15 (CD15 ${ }^{-/+}$dim vs CD15 $\left.{ }^{+++}\right) \cdot{ }^{19,20}$ Blasts of the hypogranular variant form of APL (M3v) frequently coexpress the T lineageaffiliated marker CD2 with myeloid markers CD13 and CD33. ${ }^{24-26}$

Confirmation of genetic diagnosis is mandatory and should be performed, if possible, on leukemia cells from bone marrow (BM). The identification of the APL-specific genetic lesion in leukemic cells is feasible at chromosome, DNA, RNA, and protein levels with the use of conventional karyotyping, fluorescence in situ hybridization (FISH), reverse transcriptase polymerase chain reaction (RT-PCR), or anti-PML monoclonal antibodies, respectively. Each of these has advantages and disadvantages.

Karyotyping. Karyotyping on G-banded metaphases obtained from BM samples is usually performed by conventional methods on direct, 24-hour, and 48-hour cultures. Although highly specific, cytogenetic analysis is expensive, very time-consuming, needs good quality metaphases (lacking in up to 20\%), and by definition fails to detect cases where the PML-RARA fusion results from cryptic rearrangements (false negatives). In addition, secondary chromosomal abnormalities seem not to have significant prognostic value in APL. ${ }^{27,28}$ However, cytogenetics is potentially useful in the characterization of cases lacking the $P M L-R A R A$ fusion. This may facilitate identification of rarer molecular subtypes of APL including those with $\mathrm{t}(11 ; 17)(\mathrm{q} 23 ; \mathrm{q} 21), \mathrm{t}(11 ; 17)(\mathrm{q} 13 ; \mathrm{q} 21)$, and $\mathrm{t}(5 ; 17)(\mathrm{q} 35 ; \mathrm{q} 21)$, leading to PLZF-RARA, ${ }^{29} N u M A-R A R A^{30}$ and NPM1-RARA ${ }^{31}$ fusions, respectively, as well as others more recently described. ${ }^{32-34}$

FISH analysis. FISH analysis of PML/RARA can be carried out using standard methods and commercially available fluorescently labeled probes. Although in some cases PB samples are suitable for study (particularly when hyperleukocytosis is present at diagnosis), FISH is preferably performed in BM samples. The protocol for FISH detection of $P M L / R A R A$ has been reported in detail by Grimwade et al. ${ }^{35}$ This methodology is highly specific and sensitive, and much less expensive and time-consuming than karyotyping. However, it is important to recognize the potential limitations of some probe sets used for molecular diagnostics. In particular, those that specifically detect the RARA-PML fusion gene on 
Table 1. Diagnostic workup and supportive care

\begin{tabular}{|c|c|c|}
\hline Recommendation & Level of evidence & Grade of recommendation \\
\hline 1.1. Once a diagnosis of APL is suspected, the disease should be managed as a medical emergency. & IV & C \\
\hline 1.2. Diagnosis should be confirmed by molecular detection of $P M L-R A R A$ fusion (or rare molecular variants). & Ila & B \\
\hline $\begin{array}{l}\text { 1.3. In addition to conventional karyotyping, FISH, and RT-PCR, immunostaining with anti-PML antibody can } \\
\text { be used for rapid diagnosis of APL. }\end{array}$ & Ila & B \\
\hline \multicolumn{3}{|l|}{ Management of coagulopathy } \\
\hline 1.4. Treatment with ATRA should be started immediately that a diagnosis of APL is suspected. & $\mathrm{lb}$ & A \\
\hline $\begin{array}{l}\text { 1.5. Liberally transfuse with fresh frozen plasma, fibrinogen, and/or cryoprecipitate and platelet } \\
\text { transfusions to maintain the fibrinogen concentration and platelet count above } 100-150 \mathrm{mg} / \mathrm{dL} \text { and } \\
30-50 \times 10^{9} / \mathrm{L} \text {, respectively. }\end{array}$ & $\mathrm{Ilb}$ & B \\
\hline $\begin{array}{l}\text { 1.6. The benefit of heparin, tranexamic acid, or other anticoagulant or antifibrinolytic therapy remains } \\
\text { questionable and should not be used routinely outside the context of clinical trials. }\end{array}$ & IV & C \\
\hline \multicolumn{3}{|l|}{ Management of hyperleukocytosis (WBC >10 $\times 10^{9} / \mathrm{L}$ ) } \\
\hline 1.7. Chemotherapy should be started without delay, even if the molecular results are still pending. & IV & C \\
\hline 1.8. Leukopheresis should be avoided due to risk of precipitating fatal hemorrhage. & III & B \\
\hline 1.9. Prophylactic steroids can be given, which may reduce the risk of APL differentiation syndrome. & IV & C \\
\hline \multicolumn{3}{|l|}{ Management of APL differentiation syndrome } \\
\hline $\begin{array}{l}\text { 1.10. Steroids ( } 10 \mathrm{mg} \text { dexamethasone intravenously bd) should be started immediately at the earliest } \\
\text { clinical suspicion of incipient APL differentiation syndrome. Once the syndrome has resolved, steroids } \\
\text { can be discontinued and ATO/ATRA recommenced. }\end{array}$ & lla & B \\
\hline $\begin{array}{l}\text { 1.11. Temporary discontinuation of differentiation therapy (ATRA or ATO) is indicated only in case of } \\
\text { severe APL differentiation syndrome. }\end{array}$ & Ila & B \\
\hline \multicolumn{3}{|l|}{ Management of treatment with ATO } \\
\hline 1.12. Treatment with ATO should be restricted to cases confirmed to be $P M L / R A R A$-positive. & $\mathrm{llb}$ & B \\
\hline $\begin{array}{l}\text { 1.13. Treatment with ATO requires careful monitoring to maintain electrolytes in the normal range, keeping } \\
\text { the serum potassium above } 4.0 \mathrm{mEq} / \mathrm{L} \text { and serum magnesium above } 1.8 \mathrm{mg} / \mathrm{dL} \text {. }\end{array}$ & IV & C \\
\hline 1.14. Treatment with ATO requires careful monitoring of the QT/QTc interval.* & IV & C \\
\hline
\end{tabular}

*If the QT (or QTc for patients with heart rate > 60 beats per minute) interval is prolonged longer than $500 \mathrm{msec}$, ATO should be withheld, the electrolytes (potassium and magnesium) repleted, and other medications that may cause prolonged QTc interval searched for and, ideally, discontinued. Once the QT/QTc returns to approximately $460 \mathrm{msec}$ and the electrolytes are repleted, ATO may be resumed.

der(17) will not show fusion signals in the presence of nonreciprocal rearrangements where $R A R A-P M L$ is deleted or where $P M L$ $R A R A$ is formed as a result of an insertion. Small PML-RARA insertions can also be missed by FISH when using very large probes; in such cases it is more appropriate to use relatively small cosmid probes. ${ }^{22}$ FISH provides no information about the isoform of $P M L / R A R A$, which is required for molecular monitoring of minimal residual disease. However, FISH can be useful in the investigation of suspected APL cases that lack a PML-RARA fusion, using $R A R A$ probes that span the breakpoint region to investigate for evidence of a $R A R A$ rearrangement, facilitating the identification of the fusion partner.

$R T-P C R$. RT-PCR analysis of PML-RARA is preferably carried out on RNA extracted from BM samples, although the fusion transcript is usually readily detectable in PB even in cases presenting with leukopenia. Standardized RT-PCR assays for detection of the PML-RARA fusion were established within the Biomed-1 Concerted Action. ${ }^{36}$ RT-PCR probably provides the "gold standard" approach for confirming a diagnosis of APL. In addition to its high specificity and sensitivity, it is essential for defining $P M L$ breakpoint location thereby establishing the target for reliable monitoring of MRD. However, poor RNA yield (false negative), contamination and artifacts (false positives), and the relatively long turnaround time ( $\sim 2$ days) are the main drawbacks of this methodology. In addition, it is advisable that diagnostic and monitoring samples be analyzed in reference laboratories by well-trained personnel with considerable expertise in RT-PCR for PML-RARA.

Immunostaining with anti-PML monoclonal antibodies on dry smears of BM or PB (providing circulating blasts are present) is helpful to achieve a rapid diagnosis. This technique is highly specific for presence of an underlying PML-RAR $\alpha$ fusion pro- tein, ${ }^{37-40}$ indicated by a microspeckled staining pattern $(>30$ nuclear dots) in the nuclei of leukemic cells with the PML antibody, which detects both PML-RAR $\alpha$ and the normal PML protein. The test will also be positive in those rare cases where atypical breakpoints occur within the PML locus, which could potentially be missed by standard PCR primers. In normal cells and blasts from other subtypes of leukemia (including APL molecular variants, eg, PLZF-RARA and NPM1-RARA) a wild-type PML staining pattern is observed with discrete nuclear dots (typically $<20 /$ nucleus) that relate to organelles known as PML nuclear bodies. ${ }^{22,23}$ Either indirect immunofluorescence or immunohistochemistry may be used. Results from the immunofluorescence assay can be achieved in only 2 hours. In light of its very convenient cost-benefit ratio, the assay is highly recommended for rapid confirmation of the diagnosis of APL and effectively identifying patients with the PML-RARA fusion, thereby predicting those likely to respond to molecularly targeted therapy with ATRA or ATO. This test is particularly valuable in small centers lacking access to a molecular diagnostics laboratory and in cases in which RNA is not available to confirm a diagnosis.

All of the aforementioned options are equally specific, but not equally reliable, methods to confirm the genetic diagnosis of APL. In particular, cytogenetics is much less efficient than the others. In terms of rapidity, specificity, and sensitivity, FISH and immunostaining with anti-PML monoclonal antibodies are highly efficient to confirm the diagnosis of APL. However, these techniques should not replace RT-PCR, which allows definition of the type of $P M L-R A R A$ isoform and the target for MRD evaluation.

Sample processing and banking. A specific recommendation for sample banking of all AML cases at diagnosis has been made in the revised criteria for AML diagnosis and outcome evaluation. ${ }^{41}$ The laboratory that will assume the responsibility for sample 
banking (recommended), confirmation of diagnosis at the genetic level, and monitoring MRD by RT-PCR, should receive samples as follows:

FISH or immunostaining For FISH or immunostaining studies, 3 to 4 BM smears and 3 to 4 PB smears at diagnosis are required. Samples can be sent at ambient temperature. Smears that are not used immediately must be stored for banking at $-20^{\circ} \mathrm{C}$, covered by aluminum paper.

PML-RARA analysis. For RNA extraction and RT-PCR analysis of $P M L-R A R A$, one $\mathrm{BM}$ aspirate vial $(2-3 \mathrm{~mL})$ and one $\mathrm{PB}$ vial (20-30 mL), both in sodium citrate or ethylenediaminetetraacetic acid (EDTA), are required. Samples should be processed within 24 hours. Isolated mononuclear cells in guanidium isothiocyanate can be stored for banking at $-20^{\circ} \mathrm{C}$.

Karyotyping and FISH. For conventional karyotyping and FISH studies, BM aspirate (1-2 mL) should be collected in heparin and dispatched at ambient temperature. Samples will be processed on arrival for these diagnostic studies and pelleted nuclei fixed with methanol and acetic acid (3:1) can be stored at $-20^{\circ} \mathrm{C}$.

Other genetic studies. Mutations in the gene encoding the fms-like tyrosine kinase 3 (FLT3) are seen more frequently in APL than in other subtypes of AML. ${ }^{42}$ However, although FLT3 mutations are associated with a higher white blood cell (WBC) count at presentation, they do not add to the specific diagnosis of APL or influence management, nor do they furnish important independent prognostic information. ${ }^{43,44}$ Therefore, the inclusion of the analysis of these mutations in the routine work up of APL is not recommended on the basis of current evidence. Although APL has a characteristic gene expression signature on microarray ${ }^{45,46}$ and this may provide a useful additional diagnostic tool in the future, for the time being this approach remains in the research arena.

\subsection{Institution of supportive measures to counteract the coagulopathy}

Intracerebral and pulmonary hemorrhages are relatively common life-threatening complications occurring while the characteristic coagulopathy of APL is active. These complications are not only the most frequent cause of death early during induction therapy but can also occur before the diagnosis of APL has been made and therapy started. Data about the proportion of patients developing such hemorrhages before starting induction therapy are extremely scarce in the literature. In general, major series have not provided details about patients considered not eligible for treatment because of poor clinical condition. The US Intergroup ${ }^{47}$ and the Programa de Estudio y Tratamiento de las Hemopatías Malignas (PETHEMA) group $^{48}$ have reported around $5 \%$ of patients considered not eligible for induction therapy due to very poor clinical condition (Eastern Cooperative Oncology Group [ECOG] performance status $>3$ ), mostly due to lethal or life-threatening hemorrhages before starting therapy. ${ }^{49}$ In this context, it is reasonable to recommend that supportive measures to counteract the coagulopathy should be instituted immediately the diagnosis of APL is considered and consist of fresh frozen plasma, fibrinogen and/or cryoprecipitate and platelet transfusions to maintain the fibrinogen concentration and platelet count above 100 to $150 \mathrm{mg} / \mathrm{dL}$ and 30 to $50 \times 10^{9} / \mathrm{L}$, respectively, which should be monitored at least once a day (more frequently if required). Such replacement therapy should continue during induction therapy until disappearance of all clinical and laboratory signs of coagulopathy. Despite a need-adapted transfusion policy under strict monitoring of the coagulopathy, patients presenting some factors have a higher risk of developing a fatal hemorrhage. These factors are the following: patients with active bleeding, ${ }^{50}$ hypofibrinogenemia $(<100 \mathrm{mg} / \mathrm{dL}),{ }^{51}$ or increased levels of fibrin degradation products or D-dimers combined with prolonged prothrombin time or activated partial thromboplastin time, ${ }^{48}$ as well as those presenting with increased $\mathrm{WBC}^{52,53}$ or peripheral blast ${ }^{48,50}$ counts, abnormal levels of creatinine, ${ }^{48}$ or poor performance status. ${ }^{51}$ Further investigation is warranted in these patients.

Central venous catheterization, lumbar puncture, and other invasive procedures (eg, bronchoscopy) should be avoided before and during remission induction therapy due to high risk of hemorrhagic complications. In addition, it has been also suggested that an exacerbation of the distinctive procoagulant state of APL may lead not only to increase the hemorrhagic risk but also to a higher incidence of thrombosis. The benefit of heparin, tranexamic acid, or other anticoagulant or antifibrinolytic therapy to attenuate the hemorrhagic risk remains questionable and these agents should not be used routinely outside the context of clinical trials. There are also case reports of the use of recombinant factor VIIa in the situation of severe life-threatening hemorrhage. ${ }^{54,55}$

\subsection{Initiation of treatment with ATRA}

Treatment with ATRA should be initiated without waiting for genetic confirmation of the diagnosis, preferably the same day that diagnosis is suspected. Although there is no evidence supporting this recommendation, it is reasonable to presume a favorable risk-benefit ratio associated with this approach; moreover, ATRA is unlikely to have any deleterious effect should genetic assessment fail to confirm the diagnosis of APL. ATRA is known to improve the biologic signs of APL coagulopathy rapidly; hence early initiation of this agent is likely to decrease the risk of severe bleeding. The supportive measures to be adopted upon institution of ATRA therapy are discussed below. Since APL treatment protocols are now specific for cases with documented PML-RARA fusion, for those presenting with low WBC count, administration of appropriate chemotherapy may be delayed until the genetic diagnosis is confirmed; however, in patients with hyperleukocytosis (eg, WBC $>10 \times 10^{9} / \mathrm{L}$ ), due to the high risk of induction death and retinoic acid syndrome, chemotherapy should be started without delay even if the molecular results are still pending (see section 5.1.2).

\section{Appropriate setting for the management of APL}

The recommendations by the British Committee for Standards in Hematology $(\mathrm{BCSH})$ in the recently published Guidelines on the management of acute myeloid leukemia in adults ${ }^{15}$ can be generally assumed for the management of APL. However, the peculiarities of APL deserve some additional comments and specific recommendations. In brief, the $\mathrm{BCSH}$ recommends that patients with AML should be managed by a multidisciplinary team serving a population of no fewer than 500000 and that acute leukemia induction therapy should only be carried out in those centers treating at least 5 patients per year with intensive chemotherapy. According to this recommendation, centers treating 5 to $10 \mathrm{AML}$ patients per year would have the opportunity of treating no more than one APL patient every 2 years. Although this limits experience of APL management at smaller centers, it is clear that optimum clinical treatment is dependent upon rapid access to diagnosis and hospital facilities with ATRA and blood products. This highlights the importance of guidelines to raise clinical awareness of the rapid 
actions needed for diagnosis and rigorous supportive care aspects to reduce the risk of induction death irrespective of the size of the treatment center.

\section{Treatment of newly diagnosed patients}

\subsection{Supportive care}

Recommendations for supportive care measures are summarized in Table 1 .

5.1.1. Specific supportive measures. Besides the specific supportive measures intended to reduce morbidity and mortality associated with the characteristic coagulopathy that were described above (section 3.2), other measures should be instituted to reduce some clinical complications typically associated with the administration of ATRA and ATO, such as the APL differentiation syndrome (formerly retinoic acid syndrome) seen with both agents, and several electrolyte abnormalities and prolongation of the QT interval which can occur with ATO therapy.

5.1.1.1. Prevention and management of APL differentiation syndrome. Physicians caring for patients with APL treated with ATRA or ATO should be aware of early symptoms or signs suggestive of the APL differentiation syndrome. Diagnosis of this syndrome should be suspected clinically in the presence of one of the following symptoms and signs: dyspnea, unexplained fever, weight gain, peripheral edema, unexplained hypotension, acute renal failure or congestive heart failure, and particularly by a chest radiograph demonstrating interstitial pulmonary infiltrates, or pleuropericardial effusion. Because of the life-threatening nature of the full-blown syndrome, specific treatment with dexamethasone at a dose of $10 \mathrm{mg}$ twice daily by intravenous injection should be started promptly at the very earliest symptom or sign. This policy is highly recommended even though none of the aforementioned signs and symptoms is pathognomonic of the syndrome, and they can be due to concurrent medical problems, such as bacteremia, sepsis, fungal infection or congestive heart failure. Temporary discontinuation of ATRA or ATO is indicated only in case of severe APL differentiation syndrome (ie, patients developing renal failure or requiring admission to the intensive care unit due to respiratory distress). Otherwise, these differentiating agents could be maintained unless progression to overt syndrome or lack of response to dexamethasone is observed. If a favorable response is obtained, dexamethasone should be maintained until complete disappearance of symptoms, and then ATRA or ATO should be resumed. While preemptive therapy with dexamethasone currently represents the standard approach to treat patients developing APL differentiation syndrome, there is at present no evidence that prophylactic corticosteroid is advantageous to reduce rates of morbidity and mortality associated with this syndrome. Nevertheless, in uncontrolled studies, a very low mortality or morbidity rate was reported as a result of differentiation syndrome after ATRA treatment when corticosteroids were administered prophylactically in patients presenting with WBC count greater than $5 \times 10^{9} / \mathrm{L} .{ }^{56,57}$

5.1.1.2. Prolonged QT interval associated with ATO. Treatment with ATO is associated with several electrolyte abnormalities and QT prolongation that can lead to a torsade de pointes-type ventricular arrhythmia, which can be fatal. ${ }^{58}$ This requires careful monitoring to maintain the serum potassium above $4.0 \mathrm{mEq} / \mathrm{L}$ and serum magnesium above $1.8 \mathrm{mg} / \mathrm{dL}$, well above the lower limit of normal. If possible, drugs that are known to prolong the QT interval should be discontinued. In patients who reach an absolute QT interval value longer than $500 \mathrm{msec}$, ATO should be withheld, the electrolytes (potassium and magnesium) repleted, and other medications that may cause prolonged QT interval searched for and ideally discontinued. The risk/benefit of continuing versus suspending arsenic therapy should be considered in any case. If syncope, rapid or irregular heartbeat develops, the patient should be hospitalized for ECG and electrolyte monitoring and ATO therapy should be temporarily discontinued. Once the QT/QTe returns to approximately $460 \mathrm{msec}$, the electrolytes are repleted, and the syncope and irregular heartbeat cease, ATO may be resumed. In addition to prolongation of the QT/QTc interval, approximately $13 \%$ of patients treated with ATO may develop hypokalemia or hyperglycemia.

5.1.2. Other supportive measures. Apart from the aforementioned specific measures, general supportive care aspects in APL, the use of antibiotics, hematopoietic growth factors, prevention of tumor lysis syndrome, and transfusion policy, including red cell, granulocyte, and platelet transfusions (once the coagulopathy is under control), do not differ from those applied in patients with other subtypes of AML. ${ }^{15}$

However, leukapheresis is not recommended as part of initial therapy for APL patients presenting with elevated WBC, because this procedure may exacerbate the coagulopathy and was associated with a high risk of induction death in one series. ${ }^{59}$ Early institution of chemotherapy in combination with ATRA accompanied by prophylactic steroids is the standard treatment approach in this life-threatening situation. In patients presenting with WBC count greater than $10 \times 10^{9} / \mathrm{L}$, chemotherapy is generally started on day 1 within a few hours of the first dose of ATRA, aiming to control the coagulopathy while reducing the risk of APL differentiation syndrome which is particularly high in these patients. On the other hand, treatment-related hyperleukocytosis, which frequently develops during induction with ATO, can be safely managed with careful observation, checking in particular for evidence of emerging APL differentiation syndrome (see section 5.1.1.1, "Prevention and management of APL differentiation syndrome"). ${ }^{60}$ Approximately $50 \%$ of patients treated with ATO develop leukocytosis, with a peak WBC count at approximately 20 days after the first dose. In the situation of marked hyperleukocytosis after ATO, administration of hydroxyurea can be considered although its clinical benefit is unclear; however, such leukocytosis resolves at a median of 10.5 days after the peak, despite continuation of ATO.

\subsection{Targeted treatment}

Recommendations for management during and after induction and consolidation therapy are summarized in Table 2.

\subsubsection{Induction therapy.}

5.2.1.1. The current standard approach. The simultaneous administration of ATRA and anthracycline-based chemotherapy is currently considered the standard induction treatment in newly diagnosed patients with APL. This combination results in extremely high antileukemic efficacy, leading to complete remission (CR) in $90 \%$ to $95 \%$ of patients and primary resistance has been only reported in just a few anecdotal cases. ${ }^{50,52,53,57}$ These data indicate that virtually all $P M L-R A R A$-positive APLs are sensitive to ATRA and anthracycline-based chemotherapy. Several other clinical trials conducted over the last decade have led to the optimization of ATRA and anthracycline-containing chemotherapy schedules.9,10,12,13 Initial studies involving ATRA monotherapy, which also resulted in high rates of $\mathrm{CR}$, highlighted the need for administering some type of consolidation chemotherapy to avoid disease relapse. Several subsequent studies and especially 2 randomized trials of the European APL group ${ }^{61}$ and the North American 
Table 2. Management during induction, consolidation therapy, and beyond

\begin{tabular}{|c|c|c|}
\hline Recommendation & Level of evidence & Grade of recommendation \\
\hline 2.1. Eligible patients should be offered entry into a clinical trial. & IV & C \\
\hline \multicolumn{3}{|l|}{ Induction therapy } \\
\hline $\begin{array}{l}\text { 2.2. Induction therapy should consist of the administration of concomitant ATRA and anthracycline-based } \\
\text { chemotherapy. }\end{array}$ & $\mathrm{lb}$ & A \\
\hline $\begin{array}{l}\text { 2.3. Standard induction therapy should not be modified based on the presence of leukemia cell characteristics } \\
\text { that have variably been considered to predict a poorer prognosis (eg, secondary chromosomal abnormalities, } \\
\text { FLT3 mutations, CD56 expression, and BCR3 PML-RARA isoform). }\end{array}$ & Ila & B \\
\hline $\begin{array}{l}\text { 2.4. ATO should be used as standard therapy in countries where pharmaceutical quality locally produced } \\
\text { arsenic compounds provide a more affordable treatment approach than ATRA plus chemotherapy. }\end{array}$ & III & B \\
\hline $\begin{array}{l}\text { 2.5. Treatment with ATRA should be continued until terminal differentiation of blasts and achievement of CR, } \\
\text { which occurs in virtually all patients after conventional ATRA + anthracycline induction schedules. }\end{array}$ & Ila & B \\
\hline $\begin{array}{l}\text { 2.6. Clinicians should refrain from making therapeutic modifications on the basis of incomplete blast maturation } \\
\text { (differentiation) detected up to } 50 \text { days or more after the start of treatment by morphology, cytogenetics, or } \\
\text { molecular assessment. }\end{array}$ & IV & C \\
\hline \multicolumn{3}{|l|}{ Consolidation therapy } \\
\hline $\begin{array}{l}\text { 2.7. Two or } 3 \text { courses of anthracycline-based chemotherapy are considered the standard approach for } \\
\text { consolidation therapy. }\end{array}$ & $\mathrm{lb}$ & A \\
\hline 2.8. The addition of ATRA to chemotherapy in consolidation seems to provide a clinical benefit. & $\mathrm{Ilb}$ & B \\
\hline $\begin{array}{l}\text { 2.9. Consolidation for high-risk patients younger than } 60 \text { years with WBC counts higher than } 10 \times 10 \% / \mathrm{L} \\
\text { should include at least one cycle of intermediate- or high-dose cytarabine. }\end{array}$ & $\mathrm{llb}$ & B \\
\hline $\begin{array}{l}\text { 2.10. ATO in consolidation should at present be restricted to investigation within clinical trials or those patients } \\
\text { considered unfit for conventional chemotherapy. }\end{array}$ & IV & C \\
\hline $\begin{array}{l}\text { 2.11. Molecular remission in the bone marrow should be assessed at completion of consolidation by RT-PCR } \\
\text { assay affording a sensitivity of at least } 1 \text { in } 10^{4} \text {. }\end{array}$ & Ila & B \\
\hline 2.12. Patients with confirmed molecular persistence should be considered for allogeneic HSCT. & IV & C \\
\hline $\begin{array}{l}\text { 2.13. For patients with molecular persistence who are not candidates for allogeneic HSCT, ATO or } \\
\text { gemtuzumab ozogamicin may be considered. }\end{array}$ & Ila & B \\
\hline \multicolumn{3}{|l|}{ Management after consolidation } \\
\hline $\begin{array}{l}\text { 2.14. Maintenance therapy should be used for patients who have received an induction and consolidation } \\
\text { treatment regimen wherein maintenance has shown a clinical benefit. }\end{array}$ & $\mathrm{lb}$ & A \\
\hline $\begin{array}{l}\text { 2.15. Because early treatment intervention in patients with evidence of MRD affords a better outcome than } \\
\text { treatment in full-blown relapse, every } 3 \text { months MRD monitoring of bone marrow should be offered to all } \\
\text { patients for up to } 3 \text { years after completion of consolidation therapy. }\end{array}$ & $\mathrm{llb}$ & B \\
\hline $\begin{array}{l}\text { 2.16. Bone marrow generally affords greater sensitivity for detection of MRD than blood and therefore is the } \\
\text { sample type of choice for MRD monitoring to guide therapy. }\end{array}$ & Ila & B \\
\hline $\begin{array}{l}\text { 2.17. For patients testing PCR-positive at any stage after completion of consolidation, it is recommended that a } \\
\text { bone marrow is repeated for MRD assessment within } 2 \text { weeks and that samples are sent to the local } \\
\text { laboratory, as well as to a reference laboratory for independent confirmation. }\end{array}$ & IV & C \\
\hline 2.18. CNS prophylaxis can be considered only for patients with hyperleukocytosis. & IV & C \\
\hline
\end{tabular}

Intergroup ${ }^{47}$ showed that patients receiving ATRA followed by chemotherapy had significantly better outcomes compared with patients treated with chemotherapy alone. In both studies, the CR and early death rates were not statistically different, but the relapse rate was significantly higher for the patients treated with chemotherapy alone. The outcomes with the sequential administration of ATRA followed by chemotherapy were subsequently improved on over the past decade when ATRA and chemotherapy were given simultaneously. This was clearly shown in a randomized study of the European APL group ${ }^{52}$ comparing the sequential versus the simultaneous ATRA plus chemotherapy schedule, and further confirmed in other large multicenter trials. ${ }^{53,57,62-65}$ Based on these studies, an evidence-based consensus has been reached to establish the combination of ATRA plus anthracycline-based chemotherapy as the current standard approach for newly diagnosed APL.

As to the type of anthracycline and whether it should be combined with other chemotherapy agents, both issues still remain controversial; at least as far as induction therapy is concerned. Comparable $\mathrm{CR}$ rates have been reported using ATRA plus daunorubicin and cytarabine ${ }^{52,66}$ and ATRA plus idarubicin alone, ${ }^{62,64}$ with no apparent advantage observed by adding other cytotoxic agents. ${ }^{53}$ The only randomized trial reported so $\operatorname{far}^{67}$ was unable to demonstrate differences in terms of $\mathrm{CR}$ and induction failure rates when comparing ATRA plus daunorubicin alone versus ATRA plus daunorubicin plus cytarabine for induction therapy. However, this study demonstrated an increased risk of relapse when cytarabine was omitted from both induction and consolidation therapy. A preliminary result of a second randomized trial has recently been reported which compared ATRA with idarubicin against ATRA with daunorubicin and cytarabine. There was no overall difference in response, relapse, or overall survival, but a small increase in deaths in remission was noted in the cytarabine-containing arm. ${ }^{68}$ With respect to the type of anthracycline, idarubicin has shown a slight survival advantage compared with daunorubicin in conjunction with cytarabine only in younger AML patients. ${ }^{69}$ In APL, no prospective studies have been conducted to assess the comparative value of both anthracyclines.

Exceptions to the use of the standard approach should be considered only for individual cases in which chemotherapy is contraindicated (eg, severe organ failure, anticoagulant therapy, patients older than 80 years), as well as in cases in which alternative options for induction therapy are dictated by socioeconomic factors or clinical trial design. However, modifications to the standard approach based on the presence of leukemia-cell characteristics that have variably been considered to predict a poorer 
prognosis (eg, secondary chromosomal abnormalities, FLT3 mutations, CD56, and BCR3 or short PML-RARA isoform) are not supported by the available data.

5.2.1.2. ATO as an alternative approach. After the successful results in the treatment of relapsed patients with APL first reported in China and then replicated in Western populations, ${ }^{70,71}$ several trials have been designed to investigate the role of ATO in front-line therapy. As far as we know, only 4 relatively small and selected series using ATO from China, ${ }^{72}$ Iran,${ }^{73}$ India, ${ }^{74}$ and M.D. Anderson Cancer Center (Houston, TX) ${ }^{75}$ have been published so far. The CR rate in these studies ranged from $86 \%$ to $95 \%$. However, it should be noted that ATO was combined with ATRA ${ }^{72,75}$ and/or chemotherapy ${ }^{72,74}$ and/or gemtuzumab ozogamicin ${ }^{75}$ in a variable proportion of patients, particularly those presenting with hyperleukocytosis.

Altogether, these promising results with ATO-based regimens indicate that appropriately designed comparisons with the standard ATRA plus anthracycline chemotherapy approach in terms of efficacy, safety, and cost-effectiveness are warranted. In the meantime, the use of ATO-based regimens should be restricted to patients included in clinical trials or for those in whom chemotherapy (especially anthracyclines) is contraindicated. Nevertheless, it should be remarked that, in countries where locally produced arsenic compounds provide a more affordable treatment approach than ATRA plus chemotherapy, arsenic-based regimens have been adopted as the standard of care. In this scenario, a cheaper ATO, produced under good quality control, would be effective in curing many APL patients.

5.2.1.3. Assessment of induction response. Results of morphologic, cytogenetic, and molecular evaluation should be cautiously interpreted at the end of induction therapy. Morphologic features in BM during differentiation therapy can lead to erroneously labeling some patients as resistant by inexperienced pathologists. In fact, potentially misleading cytomorphologic features due to incomplete blast maturation are occasionally seen even after several weeks from treatment initiation (up to 40-50 days). Similarly, a delayed differentiation of blasts can lead to the detection of cells displaying the $\mathrm{t}(15 ; 17)$ by conventional cytogenetics or FISH, particularly when these tests are performed at an early time after induction. These morphologic and cytogenetic assessments should not lead to any treatment modification. Rather, treatment with ATRA should be continued allowing sufficient time for terminal differentiation of blasts to occur. As discussed above, CR is attained in virtually all patients with genetically proven $P M L-R A R A$ APL. In this respect, a recent study by the PETHEMA group ${ }^{48}$ found that 6 of the 7 patients who were registered by their physicians as having primary resistant disease among 739 patients treated with ATRA plus idarubicin had been assessed for response much earlier than is currently recommended (ie, while still pancytopenic, 18-33 days after chemotherapy). ${ }^{8,41}$ Unfortunately, the premature administration of salvage therapy to these patients precluded the possibility to determine whether they genuinely exhibited primary resistance or whether in these instances there was merely a delay in terminal differentiation of APL blasts. In case of any doubt about the achievement of $\mathrm{CR}$, it is recommended to repeat another BM assessment after an additional interval of 2 to 3 weeks while keeping the patient on ATRA, and meanwhile to refrain from new therapeutic interventions. Since the introduction of this strategy, no cases of resistant leukemia have been recorded among the last 350 patients enrolled in the PETHEMA studies. ${ }^{48}$

Molecular assessment by RT-PCR on regeneration after induction has no clinical relevance, because PCR positivity at this early time point may simply reflect delayed maturation instead of resistance. Therefore, clinicians should refrain from making therapeutic decisions on the basis of results at this time point. In sharp contrast, results of PCR analyses performed after completion of consolidation are relevant to determine the relapse risk in the individual patient (see section 5.2.2.6).

5.2.2. Consolidation therapy. The achievement of molecular remission rates of roughly $95 \%$ in patients receiving at least 2 further cycles of anthracycline-based chemotherapy after induction has led to the adoption of this strategy as the standard for consolidation. ${ }^{8}$ However, some issues related to this phase of therapy remain controversial and will be discussed in detail.

5.2.2.1. The role of all-trans retinoic acid. The benefit provided by the addition of ATRA to chemotherapy for consolidation has not yet been demonstrated in randomized studies. Nevertheless, historical comparisons of consecutive trials carried out independently by the Gruppo Italiano Malattie Ematologiche dell'Adulto (GIMEMA) $^{76}$ and PETHEMA ${ }^{57}$ groups showed a statistically significant improvement in outcomes when ATRA at standard dose (45 mg/m $\mathrm{m}^{2}$ per day for adults; $25 \mathrm{mg} / \mathrm{m}^{2}$ per day for children) was given for 15 days in conjunction with chemotherapy, suggesting that ATRA contributes to reduction in relapse risk.

5.2.2.2. The role of cytarabine. From the first successful regimen using daunorubicin as monotherapy ${ }^{77}$ to the present, the role of cytarabine in APL has remained controversial. None of the studies conducted in the pre-ATRA era, including a randomized one, ${ }^{78}$ showed an advantage for addition of cytarabine to anthracyclines compared with using high-dose anthracyclines as single agents. With the incorporation of ATRA into most state-of-the-art regimens, the controversy about the role of cytarabine has remained unresolved. A recent randomized study by the European APL group ${ }^{67}$ reported an increased risk of relapse when cytarabine was omitted from a schedule including daunorubicin. The conclusions of this interesting study, however, should be interpreted with caution. As a matter of fact, the results of this study might have been largely dependent on the particular choice and dose of anthracycline chemotherapy used (daunorubicin at a cumulative dose of $495 \mathrm{mg} / \mathrm{m}^{2}$ ). Of note, a joint analysis of the PETHEMA and the European APL groups ${ }^{79}$ demonstrated a significantly lower cumulative incidence of relapse in patients younger than 65 years with $\mathrm{WBC}$ counts less than $10 \times 10^{9} / \mathrm{L}$ at presentation who were treated with anthracycline monochemotherapy (ie, with no cytarabine) in the PETHEMA LPA99 trial compared with patients in the best arm of the European APL 2000 trial including cytarabine. However, a trend in favor of cytarabine administration was observed in the same joint study for high-risk patients with WBC counts higher than $10 \times 10^{9} / \mathrm{L}$. A possible explanation for these discrepancies between chemotherapy regimens with or without cytarabine could be the different type and/or dosing schedule of the anthracyclines involved. Whereas the PETHEMA protocol used idarubicin (cumulative dose, $80-100 \mathrm{mg} / \mathrm{m}^{2}$ ) and mitoxantrone (cumulative dose, $50 \mathrm{mg} / \mathrm{m}^{2}$ ), the European APL protocol used daunorubicin (cumulative dose, $495 \mathrm{mg} / \mathrm{m}^{2}$ ). Indeed, a previous joint analysis of the PETHEMA and GIMEMA group studies that used identical type and dosage schedules of anthracyclines showed no difference in outcome with the addition of other chemotherapy agents, including cytarabine, in the GIMEMA group study. ${ }^{80}$ Similarly, the preliminary results of the MRC15 trial which were referred to earlier, did not show a benefit for the cytarabine containing arm irrespective of the white blood count. ${ }^{60}$ However, in keeping with results of the joint analysis of the PETHEMA and European APL groups, ${ }^{79}$ the most recent Italian study does suggest a benefit for using cytarabine in combination with ATRA in 
consolidation in patients with high-risk disease (ie, presenting WBC $\left.>10 \times 10^{9} / \mathrm{L}\right){ }^{76}$ Therefore, in summary, the majority of studies suggest a potential benefit in terms of reduction of relapse risk for the addition of at least one cycle of intermediate or high-dose cytarabine in patients younger than 60 years with WBC counts higher than $10 \times 10^{9} / \mathrm{L}$ but no difference in overall survival.

5.2.2.3. The role of ATO. The role of ATO in postinduction therapy for newly diagnosed APL patients has been explored not only to consolidate $\mathrm{CR}$ aiming to minimize or even eliminate chemotherapy, ${ }^{72-75}$ but also to reinforce standard ATRA plus chemotherapy regimens. Results of the 4 studies using ATO for induction and postremission therapy showed a high antileukemic activity of this agent. However, as discussed above, the use ATO in front-line therapy should at present be restricted to investigation within clinical trials or the treatment of those patients considered unfit for conventional chemotherapy. Recently, the use of ATO to reinforce standard ATRA plus chemotherapy regimens has been supported by a large randomized study by the US Intergroup. ${ }^{66}$ In this study, patients receiving 2 courses of 25 days of ATO ( 5 days a week for 5 weeks) immediately after the patient entered a CR and before the standard postremission regimen with 2 more courses of ATRA plus daunorubicin had a significantly better event-free and overall survival than those who received only ATRA plus chemotherapy. This interesting study, although providing high-level evidence for the use of ATO in consolidation, does not definitively clarify whether reinforcement of consolidation therapy with this agent improves the outcome of a given "standard therapy," because overall survival in the control arm was relatively low compared with rates reported by other groups using ATRA and anthracycline chemotherapy-based schedules. The results in the pediatric age group in the US Intergroup study were particularly disappointing compared with other standard approaches. ${ }^{81-84}$ Future studies will address whether ATO can allow deintensification of APL therapy without compromising cure rates or indeed improve on outcomes currently achieved with optimal ATRA and anthracycline-based protocols.

5.2.2.4. The role of hematopoietic stem cell transplantation. The role of hematopoietic stem cell transplantation (HSCT) in front-line therapy of APL has changed dramatically during recent years. In fact, the high cure rate obtained using upfront ATRA and chemotherapy indicates that there is no role for routine use of HSCT for patients who are in the first molecular remission at the end of consolidation. For the small fraction of patients with persistent MRD at this time point, given the poor prognosis of this subset of patients, ${ }^{85}$ allogeneic HSCT should be considered for those with a suitable human leukocyte antigen (HLA)-matched donor available. Because this group can progress rapidly to overt relapse, additional therapy (eg, ATO) can be used to reduce disease burden and ideally achieve molecular CR before transplantation. For the time being, nearly all experience in HSCT has been based on the use of ablative conditioning regimens. Data after reducedintensity conditioning in this disease are currently lacking. For those who are not candidates for allogeneic HSCT because a suitable HLA-matched donor is not available or the overall performance and clinical condition render the patient unsuitable for transplantation, other experimental options such as ATO, gemtuzumab ozogamicin, or both should be considered. Provided that the patient achieves molecular CR in the marrow and has a PCR negative harvest autologous HSCT can be considered as consolidation therapy. Although good results have been achieved using this approach, ${ }^{86,87}$ the role of transplant is uncertain, since it is possible that long-term remissions can also be achieved with multiple courses of ATO and/or gemtuzumab ozogamicin.

5.2.2.5. Risk-adapted consolidation. It is generally assumed that older patients are more vulnerable to chemotherapy toxicity and most protocols limit the age to receive full-dose chemotherapy not only for consolidation, but also for induction. Even with therapeutic approaches that have been demonstrated to have a relatively low toxicity, such as the PETHEMA treatment schedules, mortality rate in CR ranged from less than $1 \%$ in patients younger than 60 to $19 \%$ in patients older than 70 years. ${ }^{88}$ Therefore, it is a reasonable option to design therapeutic strategies to reduce treatmentrelated morbidity and mortality in this setting. In addition, there is a tendency to design risk adapted strategies to modulate treatment intensity in consolidation according to predefined risk factors for relapse, particularly presenting WBC. ${ }^{80}$ This tailored strategy seems to be an efficient approach to minimize toxicity while targeting more intensive therapy to those patients at most risk of relapse.

5.2.2.6. Molecular assessment at the end of consolidation and beyond. In contrast to the lack of clinical value of molecular assessment performed at the end of induction (see section 5.2.1.3), RT-PCR of PML-RARA in BM carried out on regeneration after the final course of chemotherapy is extremely relevant to determine the relapse risk in the individual patient. ${ }^{23,89}$ The achievement of molecular remission at this time point represents a therapeutic milestone and is considered a major treatment objective as recommended by the International Working Group for the standardization of response criteria in AML. ${ }^{41}$ The evidence supporting this statement was obtained using conventional nested RT-PCR assays, which afford a relatively low sensitivity, typically detecting 1 leukemic cell in $10^{3}$ to $10^{4}$ cells in BM. Real-time quantitative PCR (RQ-PCR) assays are marginally more sensitive and provide several advantages. ${ }^{90}$ In particular, they are less prone to contamination, allow kinetics of disease response and relapse to be defined, and enable poor quality samples that could have given rise to "false negative" results according to conventional RT-PCR assays to be identified based upon the level of endogenous control gene transcripts (eg, $A B L) .{ }^{89}$ For patients testing PCR-positive at any stage after completion of consolidation, it is recommended that a $\mathrm{BM}$ is repeated for MRD assessment within 2 weeks and that samples are sent to the local laboratory, as well as to a reference laboratory for independent confirmation. It is now accepted that patients with persistent or recurrent disease at the molecular level confirmed in 2 consecutive low-sensitivity assays after completion of consolidation will invariably relapse unless additional therapy is given. ${ }^{85,91}$ The optimal management for patients with documented molecular relapse is uncertain and has been already discussed in section 5.2.2.4, "The role of hematopoietic stem cell transplantation."

5.2.3. Maintenance therapy. Despite the benefit provided by ATRA-based maintenance therapy in 2 randomized studies, ${ }^{47,52}$ the systematic use of postconsolidation therapy is still a controversial matter in patients achieving molecular remission at the end of consolidation. As the molecular status at the end of consolidation had not been tested in these studies, it might be possible that a proportion of patients with levels of residual disease within the PCR-detectable range benefited from maintenance. Two other randomized studies, however, have recently reported no benefit from 2 different maintenance regimens. ${ }^{92,93}$ It should be noted, however, that these studies were carried out in patients testing negative for $P M L / R A R A$ at the end of consolidation. The Italian study involving ATRA, 6-mercaptopurine, and methotrexate has been published only in abstract form to date ${ }^{92}$ and the Japanese study, ${ }^{93}$ using 6 courses of intensified maintenance chemotherapy 
Table 3. Management of special situations

\begin{tabular}{|c|c|c|}
\hline Recommendation & Level of evidence & Grade of recommendation \\
\hline \multicolumn{3}{|l|}{ Older patients } \\
\hline $\begin{array}{l}\text { 3.1. Elderly patients in good clinical condition should be managed with a treatment approach similar to that } \\
\text { used in younger patients slightly attenuated in dose intensity. }\end{array}$ & Ila & B \\
\hline \multicolumn{3}{|l|}{ Patients with severe comorbidities } \\
\hline $\begin{array}{l}\text { 3.2. Older and younger patients with severe comorbidities unfit for chemotherapy (especially } \\
\text { anthracyclines) are candidates to receive ATO-based treatment schedules. }\end{array}$ & III & B \\
\hline \multicolumn{3}{|l|}{ Children } \\
\hline 3.3. ATRA at $25 \mathrm{mg} / \mathrm{m}^{2}$ per day is the recommended dose in children and adolescents. & Ila & B \\
\hline \multicolumn{3}{|l|}{ Pregnant women } \\
\hline $\begin{array}{l}\text { 3.4. Management of APL in pregnancy requires the involvement of the patient, hematologist, obstetrician, } \\
\text { and neonatologist. }\end{array}$ & III & B \\
\hline $\begin{array}{l}\text { 3.5. Retinoids are highly teratogenic and should be avoided in the first trimester unless the patient decides } \\
\text { to have a termination of pregnancy. }\end{array}$ & III & B \\
\hline 3.6. ATRA can be used in the second and third trimesters of pregnancy. & III & B \\
\hline 3.7. Arsenic derivatives are highly embryotoxic and are contraindicated at any stage of pregnancy. & IV & C \\
\hline $\begin{array}{l}\text { 3.8. In patients presenting in the first trimester and not wishing to have a termination of pregnancy, } \\
\text { induction therapy with daunorubicin alone can be offered. }\end{array}$ & IV & C \\
\hline $\begin{array}{l}\text { 3.9. Although chemotherapy appears reasonably safe in the second and third trimesters of pregnancy, it is } \\
\text { associated with an increased risk of abortions and premature delivery, and induction of labor between } \\
\text { cycles of chemotherapy should be considered. }\end{array}$ & III & B \\
\hline $\begin{array}{l}\text { 3.10. Stringent fetal monitoring, with particular emphasis on cardiac function, is recommended for patients } \\
\text { receiving ATRA with or without chemotherapy during pregnancy. }\end{array}$ & IV & C \\
\hline $\begin{array}{l}\text { 3.11. For deliveries before } 36 \text { weeks of gestation, antenatal corticosteroids before preterm delivery are } \\
\text { recommended to reduce the risk of morbidity and mortality associated with respiratory distress } \\
\text { syndrome. }\end{array}$ & $\mathrm{llb}$ & B \\
\hline 3.12. After successful delivery, breastfeeding is contraindicated if chemotherapy or ATO is needed. & IV & C \\
\hline $\begin{array}{l}\text { 3.13. Female patients with APL should be advised against conceiving while exposed to ATRA or ATO for } \\
\text { consolidation and maintenance therapy. }\end{array}$ & IV & C \\
\hline \multicolumn{3}{|l|}{ Management of therapy-related APL } \\
\hline $\begin{array}{l}\text { 3.14. Patients with tAPL should the treated like those with de novo APL, but modifications may be } \\
\text { necessary taking into account cardiac toxicity and prior anthracycline exposure. }\end{array}$ & III & B \\
\hline
\end{tabular}

without ATRA, showed no benefit in terms of reducing relapse rate and moreover was associated with a significantly poorer chance of survival. These conflicting data indicate that the relative benefit of maintenance depends upon the prior induction and consolidation therapy. Therefore, it is appropriate to use maintenance in conjunction with protocols in which it has been shown to confer benefit.

Despite the uncertainty of the benefit provided by maintenance therapy, it is a fact that several patients PCR-negative at the end of consolidation will ultimately relapse, especially among those presenting with WBC count higher than $10 \times 10^{9} / \mathrm{L}$. Previous studies have shown that the majority of these patients can be identified by sequential MRD monitoring using qualitative or quantitative PCR, allowing the opportunity for preemptive therapy to prevent disease progression to overt morphologic relapse. ${ }^{90,94,95}$

\subsection{Central nervous system prophylaxis}

The central nervous system (CNS) is the commonest site of extramedullary disease in APL and at least $10 \%$ of hematologic relapses are accompanied by CNS involvement. ${ }^{96}$ Therefore, the possibility of CNS disease should be considered in any APL patient with neurologic symptoms and be excluded in patients subject to relapse.

It was suggested, early in the ATRA era, that there was a possible association between development of extramedullary disease and the use of ATRA. However, a large study of the GIMEMA, carried out in patients treated with or without ATRA, failed to demonstrate this correlation. ${ }^{97}$ Rather, it is conceivable that extramedullary relapses are more apparent, given the greater availability of molecularly targeted therapies and prolonged sur- vival of patients. Because the majority of CNS relapses occur in patients presenting with hyperleukocytosis, ${ }^{98}$ some strategies include CNS prophylaxis for patients in this particular high-risk setting. For such patients, it is advisable to postpone CNS prophylaxis until after the achievement of CR because lumbar puncture at presentation and during induction is extremely hazardous. However, the benefit of this policy has not been established. For patients without hyperleukocytosis, in whom the risk of CNS relapse is extremely low, there is a general consensus to avoid CNS prophylaxis.

\section{Management of special situations}

Recommendations for the management of special situations are summarized in Table 3.

\subsection{Older patients}

Unlike other forms of AML arising in older patients, APL is relatively uncommon in this age group and has a relatively favorable outcome. In fact, older patients with APL seem at least as responsive to therapy as do younger patients. In addition, older patients are more likely to present with low-risk features compared with younger patients. ${ }^{88}$ To some extent, this observation may explain the relatively low relapse rate observed in patients more than 70 years of age receiving ATRA and moderately reduced anthracycline-based chemotherapy. ${ }^{88,99}$ However, as mentioned in section 5.2.2.5 ("Risk-adapted consolidation"), it is generally assumed that older patients are more vulnerable to therapy-related 
toxicity (higher rates of neutropenic sepsis and increased treatmentrelated mortality). Even with therapeutic approaches that have demonstrated a relatively low toxicity, such as the PETHEMA protocols, mortality rate in CR ranged from less than $1 \%$ in patients younger than 60 to $19 \%$ in patients older than 70 years. ${ }^{88}$ Therefore, it is reasonable to design therapeutic strategies aiming to reduce morbidity and mortality due to chemotherapy in this setting and very especially for those unfit for more intensive therapy. For the frail patients who are considered unfit for conventional treatment, ATO with or without ATRA might be a reasonable alternative to the standard ATRA plus chemotherapy approach, although supporting scientific data currently are limited, particularly with respect to rates of remission and complications such as APL differentiation syndrome. ${ }^{75}$

\subsection{Patients with severe comorbidities}

Several alternative front-line treatment approaches have been designed to minimize the use of chemotherapy in APL. Most of these are based on the use of ATRA, ATO, and gemtuzumab ozogamicin, with minimal or no chemotherapy ${ }^{75}$ (see section 5.2.1, "Induction therapy"). Although information on long-term outcome using these alternative approaches in unfit patients is lacking, they could reasonably be used for older and younger patients with severe comorbidities in which intensive chemotherapy is contraindicated (eg, patients with cardiac impairment or other severe organ dysfunction). As with conventional ATRA and anthracycline-based chemotherapy, the aim of treatment in such patients should be to achieve molecular remission, with MRD monitoring being used to guide the need for additional therapy.

\subsection{Children}

APL accounts for $4 \%$ to $8 \%$ of pediatric AML in the United States. ${ }^{100}$ Higher proportions of APL in pediatric series have been reported from other areas of the world, especially from Italy and Central and South America, ${ }^{101-103}$ but most of these studies share many methodologic limitations and deserve additional investigation. Compared with the disease in adults, APL diagnosed in childhood more frequently presents with hyperleukocytosis (approximately $40 \%$ in children vs $20 \%-25 \%$ in adults). ${ }^{88}$ Information on the long-term health status of children treated with ATRA and anthracycline-based chemotherapy is still scarce. As far as we know, only 2 relatively small pediatric series from the GermanAustrian-Swiss group ${ }^{81}$ and the European APL group, ${ }^{82}$ as well as 2 larger series from the GIMEMA ${ }^{83}$ and PETHEMA ${ }^{84}$ groups have published therapeutic results using a standard approach. Due to concern regarding the use of high dose of anthracyclines and their potential long-term cardiac toxicity, some attempts to simply reduce the exposure to these agents without any additional treatment modification have been made. In this respect, the preliminary analysis of the US Intergroup study of children treated with ATRA and reduced dose intensity of anthracycline-based chemotherapy have not been encouraging. ${ }^{66}$ It is conceivable that, given the benefit demonstrated by the addition of ATO in adults, the same therapeutic measure may compensate for a relatively low dose intensity of chemotherapy in children. ${ }^{66}$ Although this strategy has not been tested formally, ATO appears to be effective in pediatric APL as well. ${ }^{104}$ Whereas in adults several attempts have been made to use ATO-based regimens to reduce chemotherapy, as far as we know, there is only very limited experience of this agent in children with newly diagnosed APL. ${ }^{105}$
Although some protocols include CNS prophylaxis, at least for patients with hyperleukocytosis, it should be noted that neither an increased incidence of CNS relapse nor a benefit of CNS prophylaxis has been established in children.

To decrease the frequency of side effects associated with induction therapy including ATRA, particularly severe headache and pseudotumor cerebri (PTC), which are both frequently observed in children, most investigators have used a reduced dose of ATRA (eg, $25 \mathrm{mg} / \mathrm{m}^{2}$ instead of $45 \mathrm{mg} / \mathrm{m}^{2}$ ) in the pediatric age group. ${ }^{81-84}$ In this respect, the study by Castaigne et al ${ }^{106}$ showed no difference in terms of pharmacokinetics, therapeutic efficacy, triggering of hyperleukocytosis, or retinoic acid syndrome with ATRA at $25 \mathrm{mg} / \mathrm{m}^{2}$ per day compared with the standard dose of $45 \mathrm{mg} / \mathrm{m}^{2}$ per day. The apparently lower incidence of PTC and headache, together with the excellent therapeutic results obtained with this reduced dose, suggests that $25 \mathrm{mg} / \mathrm{m}^{2}$ per day is recommended as the standard dose in children and adolescents (see section 5.2.1, "Induction therapy"). Because headache is common during ATRA therapy, it is important to distinguish its etiology including PTC, CNS leukemia, or bleeding. The diagnosis of PTC is based on increased intracranial pressure with normal cerebrospinal fluid (CSF) composition and negative cerebral imaging studies (ie, computed tomography or magnetic resonance imaging scan). It is usually accompanied by papilledema, but this is not a requirement for the diagnosis of PTC. ${ }^{107}$ In this situation, sustained elevations in CSF pressure should be documented through successive lumbar punctures or by prolonged intracranial pressure monitoring, if necessary. ${ }^{108}$ Sometimes, the symptoms of PTC resolve with the initial "diagnostic" lumbar puncture. If this occurs, no further medical treatment is required. If symptoms persist, temporary discontinuation or dose reduction of ATRA, analgesics, and administration of steroids and acetazolamide are the mainstays of the medical treatment of PTC. Acetazolamide is administered in initial doses of $25 \mathrm{mg} / \mathrm{kg}$ per day and the dose titrated upward until clinical response is attained (maximum dose $100 \mathrm{mg} / \mathrm{kg}$ per day). Electrolytes must be monitored to evaluate for the development of hypokalemia and acidosis. If acetazolamide is ineffective, then prednisone can be given at a dose of $2 \mathrm{mg} / \mathrm{kg}$ per day for 2 weeks followed by a 2 -week taper. ${ }^{109}$

\subsection{Pregnant women}

The incidence of APL during pregnancy is not well established and most published data are based on case reports or very small series, but it is accepted that it is infrequent. When it occurs, it is a challenging situation, that nevertheless carries a high chance of successful outcome for mother and baby after a decision-making process that requires the involvement of the patient, hematologist, obstetrician, and neonatologist, as was recently emphasized in the guidelines on the management of AML in the United Kingdom. ${ }^{15}$ It is important to highlight that any delay in starting treatment can compromise the chance of successful remission in AML; this is particularly true in APL, which is considered a medical emergency. Apart from other considerations, given the teratogenic potential of chemotherapy, ATRA, and ATO on the fetus, the most important factors in helping to come to a decision are the gestational age and the attitude of the patient to risk, both for herself and the fetus. For practical purposes, in these guidelines we will address separately the management of APL in pregnant women presenting during the first trimester from disease arising during the second or third trimester. 
6.4.1. Management of APL during the first trimester of pregnancy. Given the teratogenic potential of chemotherapy, ATRA and arsenic trioxide on the fetus, therapeutic options for patients diagnosed during the first trimester are extremely limited in terms of the chance of successful outcome for the fetus. Although specific information regarding teratogenicity of ATRA is lacking, this agent is considered highly teratogenic, certainly because this side effect has been clearly demonstrated with other retinoids and particularly with the closely related isotretinoin. ${ }^{110}$ As a result, ATRA should be avoided during the first trimester. Administration of chemotherapy during the first trimester, although frequently safe, is also associated with fetal malformations, increased risk of abortion, and low birth weight. ${ }^{111}$ Therefore, the crucial decision in a patient with APL during the first trimester is whether to continue with the pregnancy and receive anthracycline chemotherapy alone or commit to terminate the pregnancy once the patient is hemodynamically stable, in which case conventional treatment with ATRA and chemotherapy can be commenced immediately. If elective abortion is unacceptable, administration of chemotherapy alone, without ATRA, would be the only reasonable option that can be offered during the first trimester. There is some limited evidence that idarubicin, which is more lipophilic than other anthracyclines, favoring increased placental transfer, might be more toxic in pregnancy. ${ }^{112}$ For this reason, it has been suggested that daunorubicin might be preferred because this agent is known to be effective in APL and there is more published experience of its use in pregnancy. ${ }^{111}$ It should be noted that in case of using chemotherapy alone, there will be an increased risk of hemorrhage due to release of procoagulants and plasminogen activators from malignant promyelocytes. If remission is achieved with chemotherapy and the pregnancy is progressing normally, treatment with ATRA could be administered later during the second and third trimesters. The administration of additional courses of chemotherapy during the second and third trimesters will be discussed below.

Regarding ATO, given its high potential embryotoxicity, it cannot be recommended for use at any stage of pregnancy. In fact, as far as we know, this agent has not been used in pregnant humans with APL, probably because it has been shown to be highly embryotoxic in several animal models. Human data are limited to a few populations exposed to arsenic (from drinking water or from working in or living near metal smelters) in which low birth weight, spontaneous abortion, and stillbirth are reported. ${ }^{113}$

Female patients with APL treated conventionally should be routinely advised against conceiving while exposed to ATRA or ATO for consolidation and maintenance therapy.

6.4.2. Management of APL during the second and third trimesters of pregnancy. Despite the limited clinical experience, treatment with ATRA and anthracycline-based chemotherapy seem reasonably safe when applied to patients with APL presenting during the second or third trimester of pregnancy. Although no serious complications have been observed in the mother, the considerable literature on the effect of chemotherapy on the developing fetus, recently reviewed by Culligan and colleagues, ${ }^{111}$ suggests that chemotherapy does not cause congenital malformation, but increases the risk of abortion, prematurity, low birth weight, neonatal neutropenia, and sepsis. For these reasons, there is reluctance to use chemotherapy before delivery. With regard to the administration of ATRA beyond the first trimester of pregnancy, it is considered relatively safe for mother and fetus. Although the experience is quite limited, there were no fetal malformations reported suggestive of retinoic acid embryopathy. ${ }^{111}$ However, because some cases of reversible fetal arrhythmias and other cardiac complications at birth have been reported, ${ }^{114,115}$ stringent fetal monitoring, with particular emphasis on cardiac function, is recommended for patients receiving ATRA alone or combined with chemotherapy during pregnancy.

Regarding maternal outcome after treatment with ATRAcontaining regimens, there is no evidence that this would be different in pregnancy compared with nonpregnant patients. Therefore, keeping in mind the aforementioned adverse effects on the fetus of both components of treatment, ATRA and chemotherapy, the following strategies can be outlined.

(1) Sequential use of ATRA and chemotherapy. With this approach, patients would be treated with ATRA alone until CR, delaying the administration of chemotherapy until after birth, scheduled for when the fetus is deemed to be of sufficient maturity for elective delivery. A gestational age of at least 32 weeks is considered relatively safe when appropriate neonatal care is provided. ${ }^{116}$ For deliveries before 36 weeks of gestation, antenatal corticosteroids before preterm delivery are recommended to reduce the risk of morbidity and mortality associated with respiratory distress syndrome. ${ }^{117}$ It is assumed that vaginal delivery is associated with reduced risk of bleeding, and it is preferred (if not contraindicated) to cesarean section, which might be required for other reasons. ${ }^{111}$

Regarding maternal outcomes, the expected response rate with ATRA alone is not significantly different to ATRA plus chemotherapy in terms of CR rate, but it can have an unfavorable impact on the risk of relapse. ${ }^{52}$ Perhaps, this theoretical disadvantage could be later counteracted with a reinforcement of postremission therapy. If this strategy is followed, the administration of chemotherapy should not be delayed excessively to avoid resistance and disease recurrence, which could compromise long-term outcome for the patient. It has been suggested that molecular assessment of response and subsequent RQ-PCR monitoring can be used to indicate the need for introduction of chemotherapy. ${ }^{111}$ It should also be noted that using ATRA alone there is an increased risk of APL differentiation syndrome (approximately 25\%). ${ }^{47}$

(2) Simultaneous administration of ATRA and chemotherapy. This approach, currently considered the standard treatment for patients in the nonpregnant state, provides the best chances of cure and is a clear option for high-risk patients with hyperleukocytosis and for those in which appropriate RQ-PCR monitoring is not realistic. As previously mentioned for the management of patients during the first trimester, daunorubicin might be preferred to idarubicin in pregnancy, but this is not so clear for patients with fetus in advanced gestational age.

After successful delivery, breastfeeding is contraindicated if chemotherapy or ATO is needed. The rest of management does not differ from other patients with APL.

\subsection{Therapy-related APL}

Scarce information is available about the true incidence of therapyrelated APL (tAPL) because these patients are less likely to be entered into clinical trials and available estimates are subject to important methodologic limitations being based on retrospective series $^{4,5}$ or the experience of single referral centers. ${ }^{118,119}$ In these studies, tAPL cases accounted for a range from less than $5 \%$ to $22 \%$ of all APL cases. A growing incidence of tAPL has been reported over the last few years paralleling the increased use of topoisomerase II-targeted drugs in both malignant and nonmalignant diseases. Breast carcinoma is by far the most frequent previous cancer, followed by lymphoma, with a large predominance of non-Hodgkin lymphoma compared with Hodgkin 


\begin{tabular}{l} 
Recommendation \\
\hline 4.1. For patients with confirmed molecular relapse (defined as 2 successive PCR-positive assays, with stable or \\
rising PML-RARA transcript levels detected in independent samples analyzed in 2 laboratories) preemptive \\
therapy has to be started promptly to prevent frank relapse. \\
4.2. Although ATRA in combination with chemotherapy can be used as salvage therapy, ATO-based regimens \\
are presently regarded the first option for treatment of relapsed APL. \\
4.3. Patients achieving second CR should receive intensification with SCT or chemotherapy, if possible. \\
4.4. Allogeneic HSCT is recommended for patients failing to achieve a second molecular remission. \\
4.5. Autologous HSCT is a valid option for patients without detectable MRD in the marrow and with an adequate \\
PCR negative harvest. \\
4.6. For patients in whom HSCT is not feasible, the available options include repeated cycles of ATO with or \\
without ATRA with or without chemotherapy. \\
4.7. For patients with CNS relapse, induction treatment consists of weekly triple intrathecal therapy (ITT) with \\
methotrexate, hydrocortisone, and cytarabine until complete clearance of blasts in the cerebrospinal fluid, \\
followed by 6 to 10 more spaced out ITT treatments as consolidation. Systemic treatment should also be given.
\end{tabular}

disease, whereas other tumor types were found with lower incidence. ${ }^{5}$ The drugs most commonly implicated in tAPL are epirubicin and mitoxantrone, but several cases have been reported to follow exposure to radiotherapy alone. ${ }^{6,120-122}$ Intriguingly, it has also been reported that some cases of secondary APL (sAPL) have arisen in patients whose primary tumor was treated by surgery without chemotherapy or radiotherapy exposure. ${ }^{4,5}$ The latency period between chemotherapy exposure and onset of tAPL is relatively short $(<3$ years) and typically occurs without a preceding myelodysplastic phase. Hematologic findings do not differ from those observed in de novo APL, as previously reported for other tAML with specific karyotype. ${ }^{123,124}$ However, although the incidence of secondary chromosomal abnormalities seems similar to that observed in de novo APL, the involvement of chromosomes 5,7 , or 17 appears to be more common in tAPL patients compared with those with de novo APL. ${ }^{5}$

Regarding tAPL arising in patients treated for nonmalignant diseases, it should be noted that since the approval by the US Food and Drug Administration and the European Agency for the Evaluation of Medicinal Products in 2000 of mitoxantrone for treating aggressive forms of multiple sclerosis, cases of tAPL have been increasingly reported. ${ }^{125}$ Review of published case reports indicates that APL with the $t(15 ; 17)$ is the predominant genetic subtype of tAML arising in this context, with risks of developing this complication estimated at approximately 1 in 400 patients with multiple sclerosis treated with mitoxantrone. ${ }^{126}$

Current data suggest that patients with tAPL have a relatively favorable prognosis, although the results of one study indicated a higher incidence of early death during treatment. ${ }^{5}$ Although a more precise knowledge of the outcome of patients with tAPL treated with state-of-the-art therapy should be prospectively established, at present there is generally no reason to manage these patients in a manner different from those with de novo APL. However, in a significant number of patients with tAPL, the use of conventional anthracycline-based regimens is limited by previous anthracycline exposure and/or cardiac impairment induced by treatment of the primary condition. In such situations, ATO in combination with ATRA provides an option for consolidation after standard induction therapy or as first-line treatment using schedules such as those published by the M. D. Anderson group. ${ }^{75}$

\subsection{Genetic variants of APL}

Specific management of the rare genetic variants of APL cannot be recommended because the available evidence is mostly based on single case reports. Nevertheless, because the nature of the RARA fusion partner can be critical to determine the response to ATRA and ATO, ${ }^{127}$ it is reasonable that, as a general rule, patients with ATRA-sensitive variants should be treated with standard protocols involving ATRA combined with anthracycline-based chemotherapy, while those with ATRA-resistant variants should be managed with AML-like approaches. In this regard, NuMA-RARA, NPM1-RARA, and FIP1L1-RARA are known to be ATRA-sensitive, while others are ATRA-resistant (STAT5b-RARA), ${ }^{32}$ relatively resistant (PLZF-RARA), or their sensitivity to ATRA is unknown (PRKARIA-RARA). ${ }^{33}$ Sensitivity to ATO has not been documented outside $P M L-R A R A$-positive APL, except for $P L Z F-R A R A$-positive APL, which has been shown to be resistant. ${ }^{128}$

\section{Management of relapse}

Recommendations for the management of relapse are summarized in Table 4.

\subsection{Molecular relapse}

Two studies carried out in the pre-ATO era have suggested a benefit for preemptive therapy in patients who develop molecular relapse compared with treatment initiated at the time of frank hematologic relapse. ${ }^{94,95}$ The benefit of early treatment intervention remains to be proven in the context of ATO-based relapse therapy, however the obvious risks of hemorrhagic death and development of APL differentiation syndrome when patients present with overt disease argue strongly in favor of starting therapy as early as possible in case of emergent relapse. This has provided the rationale for sequential MRD monitoring (by every 3 months BM assessment with assays achieving sensitivity of 1 in $10^{4}$ ) after completion of therapy to allow early treatment intervention. This schedule of monitoring takes into account the maximum assay sensitivities achievable for detection of APL fusion transcripts ${ }^{129}$ as well as the typical kinetics of disease relapse. ${ }^{130}$ Because the majority of relapses occur within the first 3 years after completion of consolidation, ${ }^{131}$ molecular monitoring can reasonably be discontinued after this period. The clinical utility of PB as a sample source for MRD detection remains to be established; although good concordance between blood and marrow PCR results has been reported during early stages of therapy, ${ }^{132}$ analyses of paired molecular surveillance samples taken after completion of consolidation have shown an advantage for marrow, which on average affords 1.5-log greater sensitivity. ${ }^{90,130}$ Recurrence of PCR positivity typically is apparent 
first in the marrow, and occasionally $P M L-R A R A$ transcripts do not become detectable in PB until time of hematologic relapse. ${ }^{90,130}$ Therefore, in some patients the monitoring of PB alone (even if performed monthly) could reduce the opportunity for successful delivery of preemptive therapy to prevent disease progression. Because the kinetics of disease relapse can be relatively rapid, ${ }^{130}$ preemptive therapy has to be started promptly to prevent frank relapse. The lack of specific data about optimal management for patients with documented molecular relapse leads us to assume a similar strategy to that recommended for patients with visible relapse (see section 7.2).

\subsection{Hematologic relapse}

Before the demonstration of the striking activity of ATO in APL, salvage therapy usually consisted of the readministration of ATRA and chemotherapy for induction, generally containing high-dose cytarabine and an anthracycline, followed by further chemotherapy and/or HSCT. ${ }^{95,133-135}$ Given the high antileukemic efficacy of ATO in relapsed patients and its relatively favorable toxicity profile, this agent is presently regarded as the best treatment option in this setting. ${ }^{10,136}$ Preliminary studies with ATO as salvage therapy were carried out by the Shanghai group in the early 1990s, ${ }^{137}$ and then subsequently replicated in a Western population in whom the disease had either relapsed or not responded after standard treatment with ATRA and/or anthracycline-based chemotherapy. ${ }^{138,139}$ Confirmation of the high and sustained efficacy of ATO in patients with relapsed/refractory APL has been provided by more recent studies. ${ }^{140-144} \mathrm{CR}$ rates in these trials were $80 \%$ to $90 \%$ and, in those studies that evaluated survival, $50 \%$ to $70 \%$ of patients were alive at 1 to 3 years. Current evidence suggests that use of at least 2 cycles of ATO results in the achievement of second molecular CR in nearly $80 \%$ of cases. ${ }^{145}$ The best consolidation strategy after ATO induced second remission is unknown; options include continued treatment with repeated cycles of ATO, the use of standard chemotherapy in combination with ATRA and/or ATO, and HSCT. While there is some evidence to suggest that treatment intensification with $\mathrm{HSCT}^{146}$ or chemotherapy ${ }^{141}$ may improve outcomes of patients achieving second remission with ATO, selection of the most appropriate treatment option, as well as the modality of HSCT, depends on a range of prognostic and logistic variables (eg, molecular status, duration of first remission, age, donor availability). There are no strict guidelines as regards the choice of autologous HSCT versus allogeneic HSCT in second CR. Autologous HSCT is obviously associated with a lower transplantation-related mortality than allogeneic HSCT and is a reasonable option in patients without detectable MRD and prolonged ( $>1$ year) first CR. Allogeneic HSCT involves a greater risk of nonrelapse mortality (transplantation-related) but offers a greater antileukemic activity due to the graft-versus-leukemia effect. Allogeneic HSCT could be recommended in patients failing to achieve a second molecular remission and for those with short first CR duration. ${ }^{145}$ For patients unfit to proceed to HSCT, the available options include repeated cycles of ATO with or without ATRA/standard chemotherapy. In addition, the antiCD33 monoclonal antibody conjugated to calicheamicin (gemtuzumab ozogamicin) appears to induce a high rate of molecular responses even as a single agent in advanced disease. ${ }^{147,148}$ Therefore, although the precise role of this agent in the management of relapsed APL remains unsettled, it should be explored further in the treatment strategy for relapsed patients.

\subsection{CNS and other extramedullary relapses}

At least 1 in 10 relapses of APL have a CNS component and therefore involvement of extramedullary sites (particularly CNS) should be considered in patients subject to molecular or hematologic relapse. ${ }^{96-98}$ Management of relapse in the CNS and other extramedullary sites in patients with APL is a challenging issue for which there is a notable lack of information. Extramedullary relapse, including in the CNS, can occur either in isolation or associated with BM involvement as a first relapse, but also after one or more hematologic relapses. Optimal management and outcome of APL patients in these different situations have not been critically assessed. Relapse at extramedullary sites occurs in up to $3 \%$ to $5 \%$ of patients and is emerging as a new therapeutic issue that would need international cooperation to prospectively evaluate treatment strategies in sufficient numbers of cases. In the meantime, it seems pragmatic to pursue an approach derived from experiences of the management of extramedullary relapse in acute lymphoblastic leukemia and other subtypes of acute myeloblastic leukemia. In this regard, induction treatment of CNS relapse would consist of weekly triple intrathecal therapy (ITT) with methotrexate, hydrocortisone, and cytarabine until complete clearance of blasts in the CSF, followed by 6 to 10 more spaced out ITT treatments as consolidation. Because CNS disease is almost invariably accompanied by hematologic or molecular relapse in the marrow, systemic treatment should also be given. The timing of this may be dictated by clinical circumstances. One approach could be to give ATO and ATRA as a nonmyeloablative treatment approach while ITT is being delivered. Chemotherapy regimens with high CNS penetrance (eg, high-dose cytarabine) have been used in this situation and in patients responding to treatment, allogeneic or autologous transplant should be the consolidation treatment of choice including appropriate craniospinal irradiation. In case of granulocytic sarcoma, wherever it is localized, radiation and intensive systemic therapy might be considered.

\section{Acknowledgments}

The authors thank Raul Ribeiro for critical review of the section addressing the management of children with APL, and Alan $\mathrm{H}$ Kadish and José Olagüe for critical review of the section addressing the management of the ATO-associated ECG abnormalities (QT prolongation).

This work was supported by the European Union Sixth Framework Programme, contract no. LSHC-CT-2004-503216 (European LeukemiaNet).

\section{Authorship}

Contribution: M.A.S. drafted the manuscript; and D.G. and F.L.-C. helped to integrate all changes and suggestions made by the rest of the authors (M.S.T., B.L., P.F., E.H.E., T.N., E.L., T.B., H.D., and A.K.B.), who also reviewed the manuscript and contributed to the final draft.

Conflict-of-interest disclosure: The authors declare no competing financial interests.

Correspondence: Miguel A. Sanz, Head of Hematology Department, University Hospital La Fe, Avenida Campanar 21, 46009 Valencia, Spain; e-mail: msanz@uv.es. 


\section{Appendix}

\section{Classification of evidence levels}

These classifications are used in Tables 1 through 4 .

Ia. Evidence obtained from meta-analysis of randomized controlled trials.

$\mathrm{Ib}$. Evidence obtained from at least one randomized controlled trial.

IIa. Evidence obtained from at least one well-designed controlled study without randomization.

IIb. Evidence obtained from at least one other type of welldesigned quasi-experimental study.

III. Evidence obtained from well-designed nonexperimental descriptive studies, such as comparative studies, correlation studies, and case studies.
IV. Evidence obtained from expert committee reports or opinions and/or clinical experiences of respected authorities.

\section{Classification of grades of recommendations}

A. Requires at least one randomized controlled trial as part of a body of literature of overall good quality and consistency addressing specific recommendation (evidence levels Ia and Ib).

B. Requires the availability of well-conducted clinical studies but no randomized clinical trials on the topic of recommendation (evidence levels IIa, Iib, and III).

C. Requires evidence obtained from expert committee reports or opinions and/or clinical experiences of respected authorities. Indicates an absence of directly applicable clinical studies of good quality (evidence level IV).

\section{References}

1. Ribeiro R, Rego R. Management of APL in developing countries: epidemiology, challenges and opportunities for international collaboration. Hematology Am Soc Hematol Educ Program. 2006; 162-168.

2. Douer $D$. The epidemiology of acute promyelocytic leukemia. Balliere's Best Pract Clin Hematol. 2003;16:357-367.

3. Vickers M, M, Jackson G, Taylor P. The incidence of acute promyelocytic leukemia appears constant over most of a human lifespan, implying only one rate limiting mutation. Leukemia. 2000; 14:722-726.

4. Pulsoni A, Pagano L, Lo Coco F, et al. Clinicobiological features and outcome of acute promyelocytic leukemia occurring as a second tumor: the GIMEMA experience. Blood. 2002;100:19721976.

5. Beaumont M, Sanz M, Carli PM, et al. Therapy related acute promyelocytic leukemia: a report on 106 cases. J Clin Oncol. 2003;21:2123-2137.

6. Mistry AR, Felix CA, Whitmarsh RJ, et al. DNA topoisomerase II in therapy-related acute promyelocytic leukemia. N Engl J Med. 2005;352:15291538

7. Matasar MJ, Ritchie EK, Consedine N, Magai C, Neugut AI. Incidence rates of acute promyelocytic leukemia among Hispanics, Blacks, Asians, and non-Hispanic whites in the United States. Eur J Cancer Prev. 2006;15:367-370.

8. Sanz MA, Tallman MS, Lo-Coco F. Tricks of the trade for the appropriate management of acute promyelocytic leukemia. Blood. 2005;105:30193025.

9. Tallman MS, Nabhan Ch Feusner JH, et al. Acute promyelocytic leukemia: evolving therapeutic strategies. Blood. 2002;99:759-767.

10. Douer D. New advances in the treatment of acute promyelocytic leukemia. Int J Hematol. 2002; 76(suppl 2):179-187.

11. Sanz MA, Martín G, Lo-Coco F. Choice of chemotherapy in induction, consolidation and maintenance in acute promyelocytic leukemia. Baillieres Best Pract Res Clin Haematol. 2003;16:433-451.

12. Ohno R, Asou N, Ohnishi K. Treatment of acute promyelocytic leukemia: strategy toward further increase of cure rate. Leukemia. 2003;17:14541463.

13. Sanz MA. Treatment of acute promyelocytic leukemia. Hematology Am Soc Hematol Educ Program. 2006:147-155.

14. National Comprehensive Cancer Network. NCCN Clinical Practice Guidelines in Oncology: Acute Myeloid Leukemia, v. 1. 2008. http://www.nccn. org. Accessed July 15, 2008.

15. Milligan DW, Grimwade D, Cullis JO, et al. Guidelines on the management of acute myeloid leu- kaemia in adults. Br J Haematol. 2006;135:450474.

16. General Guidelines for Methodologies on Research and Evaluation of Traditional Medicine. http://www.who.int/medicinedocs/. Accessed January 8, 2008.

17. Bennet JM, Catovsky D, Daniel MT, et al: Propos als for the classification of the acute leukemias: French-American-British (FAB) cooperative group. Br J Haematol. 1976;33:451-458.

18. Bennet JM, Catovsky D, Daniel MT et al. Proposed revised criteria for the classification of acute myeloid leukemia. Ann Intern Med. 1985; 103:626-629.

19. Orfao A, Ortuño F, de Santiago M, Lopez A, San Miguel JF. Immunophenotyping of acute leukemias and myelodysplastic syndromes. Cytometry. 2004;58A:62-71.

20. Orfao A, Chillon MC, Bortoluci AM, et al. The flow cytometric pattern of CD34, CD15 and CD13 expression in acute myeloblastic leukemia is highly characteristic of the presence of PML/RARalpha gene rearrangements. Haematologica. 1999;84: 405-412.

21. Allford S, Grimwade D, Langabeer S, et al. Identification of the $t(15 ; 17)$ in AML FAB types other than M3: evaluation of the role of molecular screening for the PML/RARalpha rearrangement in newly diagnosed AML. The Medical Research Council (MRC) Adult Leukaemia Working Party. Br J Haematol. 1999;105:198-207.

22. Grimwade D, Biondi A, Mozziconacci MJ, et al. Characterization of acute promyelocytic leukemia cases lacking the classic $t(15 ; 17)$ : results of the European Working Party. Groupe Francais de Cytogenetique Hematologique, Groupe de Francais d'Hematologie Cellulaire, United Kingdom Cancer Cytogenetics Group and BIOMED 1 European Community-Concerted Action "Molecular Cytogenetic Diagnosis in Haematological Malignancies." Blood. 2000;96:1297-1308.

23. Lo Coco F, Diverio D, Falini B, Biondi A, Nervi C, Pelicci PG. Genetic diagnosis and molecular monitoring in the management of acute promyelocytic leukemia. Blood. 1999;94:12-22.

24. Claxton DF, Reading CL, Nagarajan L, et al. Correlation of $\mathrm{CD} 2$ expression with $\mathrm{PML}$ gene breakpoints in patients with acute promyelocytic leukemia. Blood. 1992;80:582-586.

25. Paietta E, Goloubeva O, Neuberg D, et al. A surrogate marker profile for PML/RAR alpha expressing acute promyelocytic leukemia and the association of immunophenotypic markers with morphologic and molecular subtypes. Cytometry B Clin Cytom. 2004;59:1-9.

26. Guglielmi C, Martelli MP, Diverio D, et al. Immunophenotype of adult and childhood acute promyelocytic leukaemia: correlation with morphology, type of PML gene breakpoint and clinical outcome. A cooperative Italian study on 196 cases. Br J Haematol. 1998;102:1035-1041.

27. De Botton S, Chevret S, Sanz M, et al. Additional chromosomal abnormalities have no effect on the clinical outcome of patients with acute promyelocytic leukemia. Br J Haematol. 2000;111:801-806.

28. Hernandez JM, Martin G, Gutierrez NC, et al. Additional cytogenetic changes do not influence the outcome of patients with newly diagnosed acute promyelocytic leukemia treated with an ATRA plus anthracyclin based protocol. A report of the Spanish group PETHEMA. Haematologica. 2001; 86:807-813.

29. Chen S-J, Zelent A, Tong J-H, et al. Rearrangements of the retinoic acid receptor alpha and promyelocytic zinc finger genes resulting from $t(11$; 17)(q23; q21) in a patient with acute promyelocytic leukaemia. J Clin Invest. 1993;91:22602267.

30. Wells RA, Catzavelos C, Kamel-Reid S. Fusion of retinoic acid receptor to NUMA, the nuclear mitolic apparatus protein, by a variant translocation in acute promyelocytic leukaemia. Nat Genet. 1997;17:109-113.

31. Redner RL, Rush EA, Faas S, Rudert WA, Corey SJ. The $t(5 ; 17)$ variant of acute promyelocytic leukemia expresses a nucleophosmin-retinoic acid receptor fusion. Blood. 1996;87:882-886.

32. Arnould C, Philippe C, Bourdon V, Grégoire MJ, Berger R, Jonveaux P. The signal transducer and activator of transcription STAT5b gene is a new partner of retinoic acid receptor alpha in acute promyelocytic-like leukemia. Hum Mol Genet. 1999;8:1741-1749.

33. Catalano A, Dawson MA, Somana K, et al. The PRKAR1A gene is fused to RARA in a new variant acute promyelocytic leukemia. Blood. 2007; 110:4073-4076.

34. Kondo T, Mori A, Darmanin S, Hashino S, Tanaka $\mathrm{J}$, Asaka M. The seventh pathogenic fusion gene FIP1L1-RARA was isolated from a t(4;17)-positive acute promyelocytic leukemia. Haematologica. 2008;93:1414-1416.

35. Grimwade D, Gorman P, Duprez E, et al. Characterization of cryptic rearrangements and variant translocations in acute promyelocytic leukemia. Blood. 1997;90:4876-4885.

36. van Dongen JJM, Macintyre EA, Gabert J, et al. Standardized RT-PCR analysis of fusion gene transcripts from chromosome aberrations in acute leukemia for detection of minimal residual disesase. Leukemia. 1999;13:1901-1928.

37. Dyck JA, Warrell RP, Evans RM, Miller WH. Rapid diagnosis of acute promyelocytic leukemia by immunohistochemical localization of PML/ RAR-alpha protein. Blood,. 1995;86:862-867. 
38. Falini B, Flenghi L, Fagioli M, et al. Immunocytochemical diagnosis of acute promyelocytic leukemia (M3) with the monoclonal antibody PG-M3 (Anti-PML). Blood. 1997;90:4046-4053.

39. Villamor N, Costa D, Aymerich M, et al. Rapid diagnosis of acute promyelocytic leukemia by ana lyzing the immunocytochemical pattern of the PML protein with the monoclonal antibody PGM3. Am J Clin Pathol. 2000;114:786-792.

40. Gomis F, Sanz J, Sempere A, et al. Immunofluorescent analysis with the anti-pml monoclonal antibody (PG-M3) for rapid and accurate genetic diagnosis of acute promyelocytic leukemia. Ann Hematol. 2004;83:687-690.

41. Cheson BD, Bennett JM, Kopecky KJ, et al. Revised recommendations for the International Working Group for diagnosis, standardization of response criteria, treatment outcomes, and reporting standards for therapeutic trials in acute myeloid leukaemia. J Clin Oncol. 2003;21:46424649.

42. Kiyoi H, Naoe T. Biology, clinical relevance, and molecularly targeted therapy in acute leukemia with FLT3 mutation. Int J Hematol. 2006;83:301308.

43. Callens C, Chevret S, Cayuela JM, et al. Prognostic implication of FLT3 and Ras gene mutations in patients with acute promyelocytic leukemia (APL): a retrospective study from the European APL Group. Leukemia. 2005;19:11531160.

44. Gale RE, Hills R, Pizzey AR, et al. Relationship between FLT3 mutation status, biologic characteristics, and response to targeted therapy in acute promyelocytic leukemia. Blood. 2005;106: 3768-3776.

45. Haferlach T, Kohlmann A, Schnittger S, et al. AML $M 3$ and $A M L M 3$ variant each have a distinct gene expression signature but also share patterns different from other genetically defined $\mathrm{AML}$ subtypes. Genes Chromosomes Cancer. 2005; 43:113-127.

46. Valk PJM, Verhaak RGW, Beijen MA, et al. Prognostically useful gene-expression profiles in acute myeloid leukemia. New Engl J Med. 2004; 350:1617-1628

47. Tallman MS, Andersen JW, Schiffer CA, et al. Alltrans retinoic acid in acute promyelocytic leukemia. N Engl J Med. 1997;337:1201-1208.

48. De la Serna J, Montesinos P, Vellenga E, et al Causes and prognostic factors of remission in duction failure in patients with acute promyelocytic leukemia treated with all-trans retinoic acid and idarubicin. Blood. 2008;111:3395-3402.

49. Tallman MS, Brenner B, de la Serna J, et al. APL coagulopathy workshop, 21 January 2004, London, England. Leuk Res. 2005;29:347-351.

50. Di Bona E, Avvisati G, Castaman G, et al. Early haemorrhagic morbidity and mortality during remission induction with or without all-trans retinoic acid in acute promyelocytic leukemia. $\mathrm{Br}$ Haematol. 2000;108:689-695.

51. Yanada M, Matsushita T, Asou N, et al. Severe hemorrhagic complications during remission induction therapy for acute promyelocytic leukemia: incidence, risk factors, and influence on outcome. Eur J Haematol. 2007;78:213.

52. Fenaux $P$, Chastang $C$, Sanz MA, et al. A randomized comparison of ATRA followed by chemotherapy and ATRA plus chemotherapy, and the role of maintenance therapy in newly diagnosed acute promyelocytic leukemia. Blood. 1999;94: 1192-1200.

53. Burnett AK, Grimwade D, Solomon E, Wheatley $\mathrm{K}$, Goldstone $\mathrm{AH}$, on behalf of the MRC Adult Leukemia Working Party. Presenting white blood cell count and kinetics of molecular remission predict prognosis in acute promyelocytic leukemia treated with all-trans retinoic acid: result of the randomized MRC trial. Blood. 1999;93:41314143.
54. Zver S, Andoljsek D, Cernelc P. Effective treatment of life-threatening bleeding with recombinant activated factor VII in a patient with acute promyelocytic leukaemia. Eur J Haematol. 2004; 72:455-456.

55. Alimoghaddam K, Ghavamzadeh A, Jahani M. Use of NovoSeven for arsenic trioxide-induced bleeding in PML. Am J Hematol. 2006;81:720.

56. Wiley JS, Firkin FC. Reduction of pulmonary toxicity by prednisolone prophylaxis during all-trans retinoic acid treatment of acute promyelocytic leu kemia. Australian Leukaemia Study Group. Leukemia. 1995:9:774-778.

57. Sanz M, Martin G, Gonzalez M, et al. Risk adapted treatment of acute promyelocytic leukaemia with all-trans-retinoic acid and anthracycline monochemotherapy: a multicenter study by the PETHEMA group. Blood. 2004;103:1237-1243.

58. Barbey J, Pezzullo J, Soignet S. Effect of arsenic trioxide on QT interval in patients with advanced malignancies. J Clin Oncol. 2003;21:3609-3615.

59. Vahdat L, Maslak P, Miller WH, et al. Early mortality and the retinoic acid syndrome in acute promyelocytic leukernia: impact of leukocytosis, lowdose chemotherapy, PML/RAR-alpha isoform, and CD13 expression in patients treated with alltrans retinoic acid. Blood. 1994;84:3843-3849.

60. Camacho L, Soignet S, Chanel S, et al. Leukocytosis and the retinoic acid syndrome in patients with acute promyelocytic leukemia treated with arsenic trioxide. J Clin Oncol. 2000;18:26202625.

61. Fenaux P, Le Deley MC, Castaigne S, et al. Effect of all-trans retinoic acid in newly diagnosed acute promyelocytic leukemia. Results of a multicenter randomized trial. Blood. 1993;82:3241-3249.

62. Mandelli F, Diverio D, Avvisati G, et al. Molecular remission in PML/RARalpha-positive acute promyelocytic leukemia by combined all-trans retinoic acid and idarubicin (AIDA) therapy. Blood. 1997;90:1014-1021.

63. Asou N, Adachi K, Tamura J, et al. Analysis of prognostic factors in newly diagnosed acute promyelocytic leukemia treated with all-trans retinoic acid and chemotherapy. J Clin Oncol. 1998;16: 78-85.

64. Sanz MA, Martín G, Rayón C, et al. A modified AIDA protocol with anthracycline-based consolidation results in high antileukemic efficacy and reduced toxicity in newly diagnosed PML/RARalpha-positive acute promyelocytic leukemia. Blood. 1999;94:3015-3021

65. Lengfelder E, Reichert A, Schoch C, et al. Double induction strategy including high dose cytarabine in combination with all-trans retinoic acid: effects in patients with newly diagnosed acute promyelo cytic leukemia. Leukemia. 2000;14:1362-1370.

66. Powell BL, Moser B, Stock W, et al. Effect of consolidation with arsenic trioxide $\left(\mathrm{As}_{2} \mathrm{O}_{3}\right)$ on eventfree survival (EFS) and overall survival (OS) among patients with newly diagnosed acute promyelocytic leukemia (APL): North American Intergroup Protocol C9710. [abstract] J Clin Oncol. 2007(ASCO Meeting Abstracts Part I):2.

67. Ades L, Chevret $S$, Raffoux E, et al. Is cytarabine useful in the treatment of acute promyelocytic leukemia? Results of a randomized trial from the European Acute Promyelocytic Leukemia Group. J Clin Oncol. 2006;24:5703-5710.

68. Burnett AK, Hills RK, Grimwade D, et al. Idarubicin and ATRA is as effective as MRC chemo therapy in patients with acute promyelocytic leukaemia with lower toxicity and resource usage: preliminary results of the MRC AML15 trial. [abstract] Blood. 2007;110:181a

69. Kimby E, Nygren P, Glimelius B, for the SBU group. A systematic overview of chemotherapy effects in acute myeloid leukaemia. Acta Oncologica. 2001;40:231-252.

70. Douer D, Tallman MS. Arsenic trioxide: new clinical experience with an old medication in hemato- logical malignancies. J Clin Oncol. 2005;23:23962410.

71. Sanz MA, Lo-Coco F. Arsenic trioxide: its use in the treatment of acute promyelocytic leukemia. Am J Cancer. 2006;5:183-191.

72. Shen ZX, Shi ZZ, Fang J, et al. All-trans retinoic acid/ $/ \mathrm{As}_{2} \mathrm{O}_{3}$ combination yields a high quality remission and survival in newly diagnosed acute promyelocytic leukemia. Proc Natl Acad Sci U S A. 2004;101:5328-5335

73. Ghavamzadeh A, Alimoghaddam K, Ghaffari SH, et al. Treatment of acute promyelocytic leukemia without ATRA and/or chemotherapy. Ann Oncol. 2006;17:131-134.

74. Mathews V, George B, Lakshmi KM, et al. Singleagent arsenic trioxide in the treatment of newly diagnosed acute promyelocytic leukemia: durable remissions with minimal toxicity. Blood. 2006;107: 2627-2632.

75. Estey E, Garcia-Manero G, Ferrajoli A, et al. Use of all-trans retinoic acid plus arsenic trioxide as an alternative to chemotherapy in untreated acute promyelocytic leukemia. Blood. 2006;107:34693473

76. Lo-Coco F, Avvisati G, Vignetti M, et al. Front-line treatment of acute promyelocytic leukemia with AIDA induction followed by risk-adapted consolidation: results of the AIDA-2000 trial of the Italian GIMEMA group. [abstract] Blood. 2004;104:392a.

77. Bernard J, Weil M, Boiron M, et al. Acute promyelocytic leukemia: results of treatment by daunorubicin. Blood. 1973;41:489-496.

78. Avvisati G, Petti MC, Lo Coco F, et al. Induction therapy with idarubicin alone significantly influences event-free survival duration in patients with newly diagnosed hypergranular acute promyelocytic leukemia: final results of the GIMEMA randomized study LAP 0389 with 7 years of minimal followup. Blood. 2002;100:3141-3146.

79. Ades L, Sanz M, Chevret S, et al. Treatment of newly diagnosed acute promyelocytic leukemia (APL): a comparison of French-Belgian-Swiss and PETHEMA results. Blood. 2008;111:10781084

80. Sanz MA, Lo-Coco F, Martin G, et al. Definition of relapse risk and role of non-anthracycline drugs for consolidation in patients with acute promyelocytic leukemia: a joint study of the PETHEMA and GIMEMA cooperative groups. Blood. 2000;96: 1247-1252.

81. Mann G, Reinhardt D, Ritter J, et al. Treatment with all-trans retinoic acid in acute promyelocytic leukemia reduces early deaths in children. Ann Hematol. 2001;80:417-422.

82. de Botton S, Coiteux V, Chevret S, et al. Outcome of childhood acute promyelocytic leukemia with all-trans-retinoic acid and chemotherapy. J Clin Oncol. 2004;22:1404-1412.

83. Testi AM, Biondi A, Lo-Coco F, et al. GIMEMAAIEOP AIDA protocol for the treatment of newly diagnosed acute promyelocytic leukemia (APL) in children. Blood. 2005;106:447-453.

84. Ortega JJ, Madero L, Martín L, et al. Treatment with all-trans retinoic acid and anthracycline monochemotherapy for children with acute promyelocytic leukemia: a multicenter study by the PETHEMA group. J Clin Oncol. 2005;23:76327640 .

85. Breccia M, Diverio D, Noguera NI, et al. Clinicobiological features and outcome of acute promyelocytic leukemia patients with persistent polymerase chain reactiondetectable disease after the AIDA front-line induction and consolidation therapy. Haematologica. 2004;89:29-33.

86. Meloni G, Diverio D, Vignetti M, et al. Autologous bone marrow transplantation for acute promyelo cytic leukemia in second remission: prognostic relevance of pretransplant minimal residual disease assessment by reverse-transcription polymerase chain reaction of the PML/RAR alpha fusion gene. Blood. 1997;90:1321-1325. 
87. Roman J, Martin C, Torres A, et al. Absence of detectable PML-RAR alpha fusion transcripts in long-term remission patients after BMT for acute promyelocytic leukemia. Bone Marrow Transplant. 1997;19:679-683

88. Sanz MA, Vellenga E, Rayón C, et al. Alltrans retinoic acid and anthracycline monochemotherapy for the treatment of elderly patients with acute promyelocytic leukemia. Blood. 2004 104:3490-3493.

89. Grimwade D, Lo Coco F. Acute promyelocytic leukemia: a model for the role of molecular diagnosis and residual disease monitoring in directing treatment approach in acute myeloid leukemia. Leukemia. 2002;16:1959-1973.

90. Santamaria C, Chillon MC, Fernandez C, et al. Relapse-risk stratification in acute promyelocytic leukemia patients by PML-RAR[chempt]transcrip quantification. Haematologica. 2007;92:316-23.

91. Diverio D, Rossi V, Avvisati G, et al. Early detection of relapse by prospective reverse transcriptase-polymerase chain reaction analysis of the $\mathrm{PML} / \mathrm{RAR} \alpha$ fusion gene in patients with acute promyelocytic leukemia enrolled in the GIMEMAAIEOP multicenter "AIDA" trial. Blood. 1998;92: 784-789.

92. Avvisati G, Petti MC, Lo Coco F, et al. AIDA: The Italian way of treating acute promyelocytic leukemia (APL), final act [abstract]. Blood. 2003;102: $142 \mathrm{a}$

93. Asou N, Kishimoto Y, Kiyoi H, et al. Arandomized study with or without intensified maintenance chemotherapy in patients with acute promyelocytic leukemia who have become negative for PML-RAR transcript after consolidation therapy: The Japan Adult Leukemia Study Group (JALSG) APL97 study. Blood. 2007;110:59-66.

94. Lo Coco F, Diverio D, Avvisati G, et al. Therapy of molecular relapse in acute promyelocytic leukemia. Blood. 1999;94:2225-2229.

95. Esteve J, Escoda L, Martín G, et al. Outcome of patients with acute promyelocytic leukemia failing to front-line treatment with all-trans retinoic acid and anthracycline-based chemotherapy (PETHEMA protocols LPA96 and LPA99): benefit of an early intervention. Leukemia. 2007;21:446-452.

96. Evans GD, Grimwade DJ. Extramedullary disease in acute promyelocytic leukemia. Leuk Lymphoma. 1999;33:219-229.

97. Specchia G, Lo-Coco F, Vignetti M, et al. Extramedullary involvement at relapse in acute promyelocytic leukemia patients treated or not with ATRA: a report by the GIMEMA Group. J Clin Oncol. 2001;19:4023-4028.

98. de Botton S, Sanz MA, Chevret S, et al. Extramedullary relapse in acute promyelocytic leukemia treated with all-trans retinoic acid and chemotherapy. Leukemia. 2005;20:35-41.

99. Mandelli F, Latagliata R, Avvisati G, et al. Treatment of elderly patients ( $>$ or $=60$ years) with newly diagnosed acute promyelocytic leukaemia. Results of the Italian multicenter group GIMEMA with ATRA and idarubicin (AIDA) protocols. Leukemia. 2003;17:1085-1090.

100. Feusner JH, Gregory J. Acute promyelocytic leukemia in children. ASCO Educational Book. 2006; 577-581.

101. Maule MM, Dama E, Mosso ML, Magnani C, Pastore G, Merletti F. High incidence of acute promyelocytic leukemia in children in northwest Italy, 1980-2003: a report from the Childhood Cancer Registry of Piedmont. Leukemia. 2008; 22:439-441.

102. Malta CA, Pacheco EC, Cantu RA, et al. Childhood acute promyelocytic leukemia in Nicaragua. Ann Oncol. 1993:4:892-894.

103. Gomez SM, Schuttenberg V, Armendariz H, et al. Childhood acute leukemia: a single institution experience in La Plata, Argentina. Med Pediatr Oncol. 2001;36:383-385

104. Fox E, Razzouk BI, Widemann BC, et al Plase 1 trial and pharmacokinetic study of arsenic trioxide in children and adolescents with refractory or relapsed acute leukemia, including acute promyelocytic leukemia or lymphoma. Blood. 2008;111: 566-573.

105. George B, Mathews V, Poonkuzhali B, et al. Treatment of children with newly diagnosed acute promyelocytic leukemia with arsenic trioxide: a single center experience. Leukemia. 2004;18: 1587-1590.

106. Castaigne S, Lefebvre $P$, Chomienne $C$, et al. Effectiveness and pharmacokinetics of low-dose all-trans retinoic acid $\left(25 \mathrm{mg} / \mathrm{m}^{2}\right)$ in acute promyelocytic leukemia. Blood. 1993;82:3560-3563.

107. Wang SJ, Silberstein SD, Patterson S, et al. Idiopathic intracranial hypertension without papilledema. Neurology. 1998;51:245-249.

108. Spence JD, Amacher AL, Willis NR. Benign intracranial hypertension without papilledema: roll of 24-hour cerebrospinal fluid pressure monitoring in diagnosis and management. Neurosurgery. 1980; 7:326-336

109. Robertson WC Jr, Wilson M-CB, Baker MJ. Pseudotumor cerebri: pediatric perspective. In: Sheth RD, Talavera F, Mack KJ, Benbadis SR, Lorenzo NY, eds. eMedicine. http://www.emedicine.com/neuro/topic537.htm. Accessed February 14, 2008.

110. Lammer EJ, Chen DT, Hoar RM, et al. Retinoic acid embryopathy. N Engl J Med. 1985;313:837841.

111. Culligan DJ, Merriman L, Kell J, et al. The management of acute promyelocytic leucemia presenting during pregnancy. Clin Leukemia. 2007;1: 183-191.

112. Cardonick E, lacobucci A. Use of chemotherapy during human pregnancy. Lancet Oncol. 2004;5: 283-291.

113. U.S. Environmental Protection Agency. Arsenic compounds. http://www.epa.gov. Accessed January 8, 2008.

114. Terada Y, Shindo T, Endoh A, Watanabe M, Fukaya T, Yajima A. Fetal arrhythmia during treatment of pregnancy-associated acute promyelocytic leukemia with all-trans retinoic acid and favourable outcome. Leukemia. 1997;11:454455.

115. Siu BL, Alonzo MR, Vargo TA, et al. Trasient dilated cardiomyopathy in a newborn exposed to idarubicin and all-trans retinoic acid (ATRA) early in the second trimester of pregnancy. Int J Gynecol Cancer. 2002;12:399-402.

116. Slattery MM, Morrison JJ. Preterm delivery. Lancet. 2002;360:1489-1497.

117. Royal College of Obstetricians and Gynecologists. Antenatal corticosteroids to reduce respiratory distress syndrome. Guideline No. 7. Available at: www.rcog.org.uk/resources/Public/pdf/ Antenatal_corticosteroids_No7.pdf. Accessed April 1, 2007.

118. Pollicardo N, O'Brien S, Estey EH, et al: Secondary acute promyelocytic leukemia: characteristics and prognosis of 14 patients from a single institution. Leukemia. 1996;10:27-31.

119. Beaumont M, Lai JL, Simonnet E, et al: Therapy related acute promyelocytic leukemia (tAPL): increasing incidence, especially after nonHodgkin's lymphoma (NHL) treated intensively [abstract]? Blood. 2000;96:321a.

120. Andersen MK, Larson RA, Mauritzson N Schnittger S, Jhanwar SC, Pedersen-Bjergaard J. Balanced chromosome abnormalities inv(16) and $t(15 ; 17)$ in therapy related myelodysplastic syndromes and acute leukemia: report from an international workshop. Genes Chromosomes Cancer. 2002:33:395-400.

121. Pedersen-Bjergaard J. Insights into leukemogenesis from therapy-related leukemia. N Eng J Med. 2005;352:1591-1594.

122. Smith SM, Le Beau MM, Huo D, et al. Clinicalcytogenetic association in 306 patients with therapy-related myelodysplasia and myeloid leukemia: the University Chicago series. Blood. 2003;102:43-52

123. Pedersen-Bjergaard J, Philip P, Larsen SO, et al. Chromosome aberrations and prognostic factors in therapy-related myelodysplasia and acute nonlymphocytic leukemia. Blood. 1990;76:10831091

124. Quesnel B, Kantarjian H, Pedersen-Bjergaard J et al. Therapy-related acute myeloid leukemia with $t(8 ; 21)$, inv(16), and $t(8 ; 16)$ : a report on 25 cases and review of the literature. J Clin Oncol. 1993;11:2370-2379.

125. Ledda A, Caocci G, Spinicci G, et al. Two new cases of acute promyelocytic leukemia following mitoxantrone treatment in patients with multiple sclerosis. Leukemia. 2006;20:22172218.

126. Ghalie RG, Mauch E, Edan G, et al. A study of therapy-related acute leukaemia after mitoxantrone therapy for multiple sclerosis. Mult Scler. 2002;8:441-445.

127. Mistry AR, Pedersen EW, Solomon E, Grimwade $D$. The molecular pathogenesis of acute promyelocytic leukaemia: implications for the clinical management of the disease. Blood Rev. 2003;17: 71-97.

128. Koken MHM, Daniel MT, Gianni M, et al. Retinoic acid, but not arsenic trioxide, degrades the PLZF/ $\mathrm{RAR} \alpha$ fusion protein, without inducing terminal differentiation or apoptosis, in a RA-therapy resistant $t(11 ; 17)(q 23 ; q 21)$ APL patient. Oncogene. 1999;18:1113-1118.

129. Freeman SD, Jovanovic JV, Grimwade D. Development of minimal residual disease-directed therapy in acute myeloid leukemia. Semin Oncol. 2008;35:388-400.

130. Grimwade D, Jovanovic JV, Hills RK, et al. Prospective minimal residual disease monitoring to predict relapse of acute promyelocytic leukemia and direct pre-emptive arsenic trioxide therapy. J Clin Oncol. 2009; in press.

131. Gallagher RE, Yeap BY, Bi W, et al. Quantitative real-time RT-PCR analysis of PML-RAR mRNA in acute promyelocytic leukemia: assessment of prognostic significance in adult patients from intergroup protocol 0129. Blood. 2003;101:25212528.

132. Sanz MA, Montesinos P, Vellenga E, et al. Riskadapted treatment of acute promyelocytic leuke mia with all-trans-retinoic acid and anthracycline monochemotherapy: long-term outcome of the LPA 99 multicenter study by the PETHEMA group. Blood. Prepublished online July 29, 2008; DOI 10.1182/blood-2008-05-159632.

133. Estey EH. Treatment options for relapsed acute promyelocytic leukaemia. Best Pract Res Clin Haematol. 2003;16:521-534.

134. Castagnola C, Lunghi M, Corso A, et al. Management of acute promyelocytic leukemia relapse in the ATRA era. Haematologica. 1998; 83:714-717.

135. Thomas X, Dombret H, Cordonnier C, et al. Treatment of relapsing acute promyelocytic leukemia by all-trans retinoic acid therapy followed by timed sequential chemotherapy and stem cell transplantation. Leukemia. 2000;14:10061013.

136. Chen Z, Zhao WL, Shen ZX, et al. Arsenic trioxide and acute promyelocytic leukemia: clinical and biological. Curr Top Microbiol Immunol. 2007; 313:129-144.

137. Shen Z-X, Chen G-Q, Ni J-H, et al. Use of arsenic trioxide (As2O3) in the treatment of acute promyelocytic leukemia (APL): II. Clinical efficacy and pharmacokinetics in relapsed patients. Blood. 1997;89:3354-3360

138. Soignet SL, Maslak P, Wang Z-G, et al. Complete remission after treatment of acute promyelocytic leukemia with arsenic trioxide. N Engl J Med. 1998;339:1341-1348. 
139. Soignet SL, Frankel SR, Douer D, et al. United States multicenter study of arsenic trioxide in relapsed acute promyelocytic leukemia. J Clin Oncol. 2001;19:3852-3860.

140. Au WY, Lie AK, Chim CS, et al. Arsenic trioxide in comparison with chemotherapy and bone marrow transplantation for the treatment of relapsed acute promyelocytic leukaemia. Ann Oncol. 2003; 14:752-757

141. Lazo G, Kantarjian H, Estey E, et al. Use of arsenic trioxide (As2O3) in the treatment of patients with acute promyelocytic leukemia: the M.D. Anderson experience. Cancer. 2003;97:2218-2224.

142. Niu C, Yan H, Yu T, et al. Studies on treatment of acute promyelocytic leukemia experience with arsenic trioxide: remission induction, fol- low-up, and molecular monitoring in 11 newly diagnosed and 47 relapsed acute promyelocytic leukemia patients. Blood. 1999;94:33153324.

143. Raffoux E, Rousselot P, Poupon J, et al. Combined treatment with arsenic trioxide and all-trans retinoic acid in patients with relapsed acute promyelocytic leukemia. J Clin Oncol. 2003;21:23262334.

144. Shigeno K, Naito K, Sahara N, et al. Arsenic trioxide therapy in relapsed or refractory Japanese patients with acute promyelocytic leukemia: updated outcomes of the phase II study and postremission therapies. Int J Hematol. 2005;82:224229.

145. Tallman MS. Treatment of relapsed or refractory acute promyelocytic leukemia. Best Pract Res Clin Haematol. 2007;20:57-65.

146. Douer D, Hu W, Giralt S, et al: Arsenic trioxide (trisenox) therapy for acute promyelocytic leukemia in the setting of hematopoietic stem cell transplantation. Oncologist. 2003;8:132140.

147. Estey EH, Giles FJ, Beran M, et al. Experience with gemtuzumab ozogamicin ("mylotarg") and all-trans-retinoic acid in untreated acute promyelocytic leukemia. Blood. 2002;99:4222 4224.

148. Lo-Coco F, Cimino G, Breccia M, et al. Gemtuzumab ozogamicin (Mylotarg) as a single agent for molecularly relapsed acute promyelocytic leukemia. Blood. 2004;104:1995-1999. 